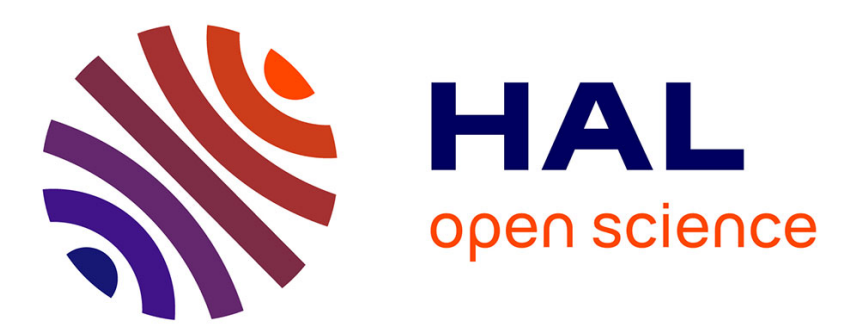

\title{
Angular MIMO for Underwater Wireless Optical Communications: Link Modelling and Tracking
} Abdallah S. Ghazy, Steve Hranilovic, Mohammad Ali A Khalighi

\section{To cite this version:}

Abdallah S. Ghazy, Steve Hranilovic, Mohammad Ali A Khalighi. Angular MIMO for Underwater Wireless Optical Communications: Link Modelling and Tracking. IEEE Journal of Oceanic Engineering, 2021, 46 (4), pp.1391-1407. 10.1109/JOE.2021.3055477 . hal-03147572

\section{HAL Id: hal-03147572 \\ https://hal.science/hal-03147572}

Submitted on 20 Feb 2021

HAL is a multi-disciplinary open access archive for the deposit and dissemination of scientific research documents, whether they are published or not. The documents may come from teaching and research institutions in France or abroad, or from public or private research centers.
L'archive ouverte pluridisciplinaire HAL, est destinée au dépôt et à la diffusion de documents scientifiques de niveau recherche, publiés ou non, émanant des établissements d'enseignement et de recherche français ou étrangers, des laboratoires publics ou privés. 


\title{
Angular MIMO for Underwater Wireless \\ Optical Communications: Link Modelling and Tracking
}

\author{
Abdallah S. Ghazy, Steve Hranilovic and Mohammad-Ali Khalighi
}

\begin{abstract}
Angular imaging multiple-input/multiple-output (A-MIMO) is investigated for short-range, highspeed underwater wireless optical communications (UWOCs) where, unlike conventional imaging MIMO (C-MIMO), data are transmitted in an angle rather than in space. In this approach, the strict requirements of on-axis alignment and fixed channel length are relaxed. This technique also allows for simpler estimation of the relative misalignment between the transmitter and the receiver from the received image. For the first time, we derive a comprehensive model for the underwater A-MIMO link by taking into account link misalignment, background noise, as well as seawater absorption and scattering. Power distributions at the receiver are modeled by the angle-of-arrival (AoA) of the received signal on the lens and its position-of-arrival (PoA) on the focal plane of the detector. We further propose and model a tracked A-MIMO (TA-MIMO) system that maintains the alignment between the two ends of the link, for which the distribution of the residual tracking error is calculated. The UWOC channel capacity is then estimated for buoyed-to-fixed (B2F) (which has dominant angular misalignments) and mobile-tofixed (M2F) (which has dominant off-axis misalignment) communication scenarios. Numerical results indicate that in the B2F scenario, A-MIMO is sensitive to angular misalignments; however, TA-MIMO outperforms C-MIMO. In the case of M2F links, A-MIMO greatly outperforms C-MIMO when off-axis misalignments are present. This work serves as a design guide to determine the selection of A-MIMO, TA-MIMO or C-MIMO receivers depending on the misalignment conditions for a particular underwater application.
\end{abstract}

Abdallah S. Ghazy and Steve Hranilovic are with the Electrical and Computer Engineering School, McMaster University, Ontario, Canada (e-mail: ghazya@mcmaster.ca, hranilovic@mcmaster.ca).

Mohammad-Ali Khalighi is with Aix-Marseille University, CNRS, Centrale Marseille, Institut Fresnel, Marseille, France (e-mail: Ali.Khalighi@fresnel.fr).

This work has been supported by the Natural Science and Engineering Research Council in Canada. This work was presented in part at the 16th Canadian Workshop on Information Theory, June 2019. 


\section{Index Terms}

Underwater wireless optical communications, angular MIMO, link misalignment, scattering, channel capacity.

\section{INTRODUCTION}

Underwater acoustic communication is an intrinsically low data-rate transmission technology, offering communication speeds on the order of kilobits/second (kbps). Underwater wireless optical communication (UWOC) is a promising candidate for high data-rate applications with rates that can approach gigabits/second (Gbps) [1]-[4]. This technology takes advantage of the directivity of optical beams, low energy consumption, and relatively small size transceivers inherent to free-space optics. However, the capacity of UWOC links is in practice limited by the high optical beam attenuation in seawater and the limited bandwidth of the opto-electronic components [5], [6]. For overcoming these problems, among the available solutions, is using multiple-input multiple-output (MIMO) techniques. The MIMO system is implemented using an array of emitters and detectors in the transmitter and receiver, respectively. By utilizing the spatial degrees of freedom, the MIMO systems increase the system capacity and reduce the peak of the transmitted optical power per the emitter. The reducing in the peak of the transmitted power mitigates the non-linearity of the emitters and increases the eye-safety. However, using MIMO architecture increase the complexity of the communication system, which is the cost of the MIMO advantages [7], [8].

In atmospheric wireless optical communications links, imaging MIMO has been proposed for high-speed short-range indoor applications [7]. A typical imaging MIMO configuration, termed here conventional MIMO (C-MIMO), consists of an array of emitters and imaging receiver. In [9], Zeng et al. evaluated the performance of imaging and non-imaging MIMO systems, showing the potential of the former in reducing spatial inter-channel interference problems. However, imaging MIMO systems require perfect alignment and compensation of changes in magnification due to channel length variations [8]. Recently, a fixed-scale imaging MIMO architecture was proposed in [10] for short-range indoor applications, with robustness against off-axis misalignment as well as fixed magnification, independent of the channel length [10].

Comparatively, little work has been done on the investigation of MIMO approaches for highspeed short range UWOC links [1]. In [11], a $2 \times 2$ non-imaging MIMO system was investigated considering the impact of inter-channel interference. However, perfect alignment and a fixed 
channel length were assumed. In [12], the capacity of a buoy node was studied focusing on the impact of misalignment induced by sea waves at different wind speeds. In a follow-up paper [13], the capacity of a $3 \times 2$ non-imaging MIMO buoyed node was investigated under turbulence, scattering and misalignment impairments. For underwater imaging MIMO systems, Li et al. [14] showed the effect of inter-spacing between laser diodes (LDs) and photo-detectors (PDs) on the performance of a $2 \times 2$ imaging C-MIMO link over $2.4 \mathrm{~m}$ under perfect alignment conditions. A common thread through these studies is that severe misalignment losses due to sea waves or currents are identified as key impairments to C-MIMO UWOC links.

Recently, Xu et al. [15] and Ghazy et al. [16] applied the fixed-scale architecture of [10] to UWOC links. In particular, the authors in [15] numerically quantified the bit-error rate (BER) performance of the fixed scale MIMO system underwater over a $1 \mathrm{~m}$ range. In [16], Ghazy et al. proposed angular MIMO (A-MIMO) using the architecture of the fixed-scale MIMO system. There in, the capacity performance of $(9 \times 9)$ A-MIMO and C-MIMO systems are contrasted under off-axis misalignment and variation of a channel length.

For the first time, we extend our work in [16] by deriving a comprehensive model and presenting a design for short-range high-capacity underwater A-MIMO systems where data are transmitted in angle rather than in space. Additionally, a tracking scheme is proposed and its performance quantified using the unique features of the A-MIMO system to yield a tracked AMIMO (TA-MIMO) system. More specifically, in contrast to [15], [16], here we derive detailed link modelling, which provides useful insight into the design of underwater A-MIMO and TAMIMO systems from both channel capacity and tracking perspectives. The main contributions of this paper are summarized as follows:

- A comprehensive model for underwater A-MIMO links is derived considering both angle of arrival (AoA) and position of arrival (PoA) distributions.

- Closed-form polar and azimuthal AoA distributions are derived for the central source at on-axis alignment conditions.

- The derived equations for AoA distributions are verified through Monte Carlo numerical ray tracing (MCNRT) simulations [17], [18].

- Based on the optical properties and geometry of the A-MIMO architecture, a new tracking scheme is proposed and modelled, termed pointing-localization-tracking (PLT) scheme, and its efficiency is demonstrated through numerical results.

- The distribution of the tracking residual errors (TRE) is calculated using MCNRT method 


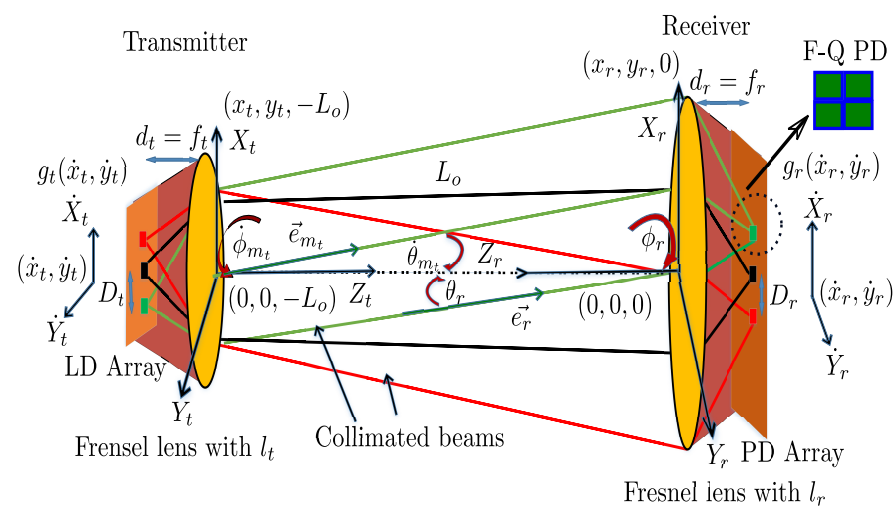

Fig. 1. Architecture of a $3 \times 3$ A-MIMO system with parameters given in Table I.

for the cases of a buoyed-to-fixed (B2F) communication model.

- For the case of B2F and mobile-to-fixed (M2F) communication scenarios, the average capacity of a $49 \times 49$ link using A-MIMO, TA-MIMO, and C-MIMO techniques, are contrasted.

The remainder of the paper is organized as follows. Section II describes the optical architecture of an A-MIMO link and its intrinsic characteristics. Section III focuses on A-MIMO link modelling, where AoA and PoA distributions are derived with closed-form expressions for special cases. Section IV presents the capacity of A-MIMO and C-MIMO links under two realistic B2F and M2F communication scenarios. In Section V, intrinsic localization features of A-MIMO systems are demonstrated. A PLT scheme is proposed and TRE distributions are computed using MCNRT. Afterwards, in Section VI, the numerical results on link modelling are presented, and the capacity of A-MIMO, TA-MIMO, and C-MIMO links are contrasted for the cases of clear and coastal seawaters. The paper concludes in Section VII with directions for future work.

Notation: In this article, vectors and matrices are denoted by bold-face lower and upper-case letters, respectively. Probability distribution functions are denoted by $p(\cdot)$. A Gaussian random variable with zero mean and $\sigma^{2}$ variance is denoted by $\mathcal{N}\left(0, \sigma^{2}\right)$. Also, the complex conjugate of $G(\cdot)$ is written as $G^{*}(\cdot)$.

\section{Angular MiMO}

Imaging MIMO systems are well suited to high-speed short-range underwater applications [14]-[16]. Practical examples of such links include buoys and autonomous underwater vehicles 


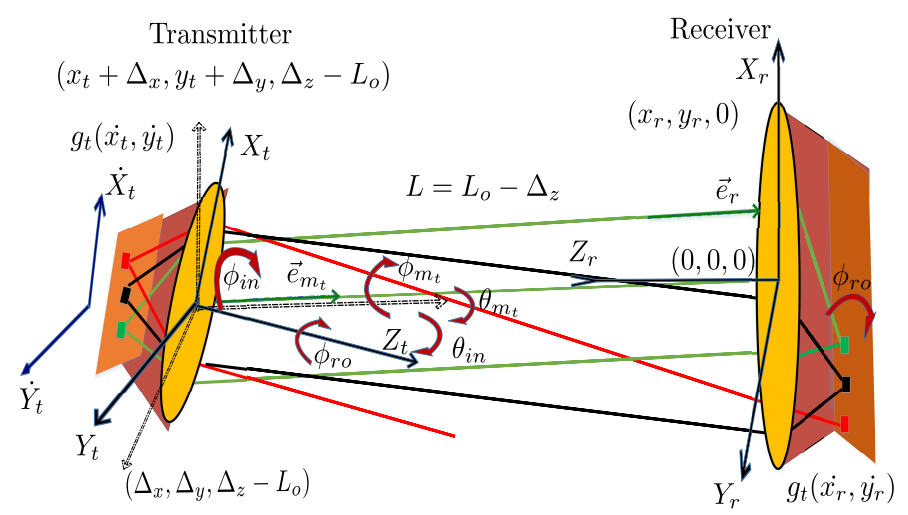

Fig. 2. Beam axes and orientations for the case of a misaligned $3 \times 3$ A-MIMO system. The definition of the different parameters are provided in Table I.

(AUVs) as will be discussed in Section IV. C-MIMO systems require perfect alignment and a fixed magnification between the two ends of the link, which is difficult to maintain in practice [15], [16]. In the following, we present the key advantages of underwater A-MIMO systems that relax these two key impairments of C-MIMO.

\section{A. Description of A-MIMO Systems}

Consider a general $\left(M_{t} \times M_{r}\right)$ A-MIMO structure. At the transmitter, $M_{t}$ LDs are used, arranged on a square array of $\left(\sqrt{M_{t}} \times \sqrt{M_{t}}\right)$, with the spacing $D_{t}$ between the centres of the LDs. The centre of the $m_{t}^{\text {th }} \mathrm{LD}, m_{t} \in\left(1,2, \ldots, M_{t}\right)$, is obtained by $\dot{x}_{m_{t}}=D_{t}\left(2 i-\left(\sqrt{M_{t}}+1\right)\right)$ and $\dot{y}_{m_{t}}=D_{t}\left(2 j-\left(\sqrt{M_{t}}+1\right)\right)$. Note that $m_{t}$ is related to $\{i, j\} \in\left(1,2, \ldots, \sqrt{M_{t}}\right)$ through $m_{t}=\left(j+(i-1) \sqrt{M_{t}}\right)$. Likewise, at the receiver, located at a nominal range of $L_{o}$, the $M_{r}$ PDs are arranged on a square array of $\left(\sqrt{M_{r}} \times \sqrt{M_{r}}\right)$, with spacing $D_{r}$ between their centers. The centre of the $m_{r}^{\text {th }} \mathrm{PD}, m_{r} \in\left(1,2, \ldots, M_{r}\right)$, is given by $\dot{x}_{m_{r}}=D_{r}\left(2 i-\left(\sqrt{M_{r}}+1\right)\right)$ and $\dot{y}_{m_{r}}=D_{r}\left(2 j-\left(\sqrt{M_{r}}+1\right)\right)$. Here, $m_{r}$ is related to $\{i, j\} \in\left(1,2, \ldots, \sqrt{M_{r}}\right)$ by $m_{r}=$ $\left(j+(i-1) \sqrt{M_{r}}\right)$. Figures 1 and 2 illustrate the system architecture of a $(3 \times 3)$ A-MIMO link for the two cases of perfectly-aligned and misaligned transmitter and receiver, respectively (we have taken $M_{t}=M_{r}=3$ just to simplify the illustration). The receiver axes $\left(X_{r}, Y_{r}, Z_{r}\right)$ are fixed at $(0,0,0)$ and considered as reference. The considered transmitter axes $\left(X_{t}, Y_{t}, Z_{t}\right)$ are then either aligned or misaligned. The definition of the different parameters, angles, etc. are summarized in Table I. 
At the transmitter, the LD array produces an image $g_{t}\left(\dot{x}_{t}, \dot{y}_{t}\right)$, where $\left(\dot{x}_{t}, \dot{y}_{t}\right)$ is the position in the plane of the LD array. Moreover, a convex lens (e.g., a Fresnel lens) with focal length $f_{t}$ and diameter $l_{t}$ is placed at a distance $d_{t}$ from the array, where $d_{t}=f_{t}$. This lens collimates the beam of the $m_{t}^{t h} \mathrm{LD}$ and transmits it in the direction of the unit vector $\vec{e}_{m_{t}}$, specified by polar and azimuthal angles $\left(\dot{\theta}_{m_{t}}, \dot{\phi}_{m_{t}}\right)$, defined with respect to the transmitter axes. For instance, the transmitted unit vector associated with the green LED is shown by a green arrow in Fig. 1. In the transmitter lens plane, each ray is sent from position $\left(x_{t}, y_{t},-L_{o}\right)$ according to the receiver axes as shown in the figure.

At the receiver side, a convex lens with focal length $f_{r}$ and diameter $l_{r}$ is placed at distance $d_{r}=f_{r}$ from the PD array. The incident ray arrivals at position $\left(x_{r}, y_{r}, 0\right)$ are shown in Fig. 1. The lens receives each beam with direction along with the unit vector $\vec{e}_{r}$, specified by polar and azimuthal angles $\left(\theta_{r}, \phi_{r}\right)$. Then, the lens projects the received image $g_{r}\left(\dot{x}_{r}, \dot{y}_{r}\right)$ on the PD array, i.e., the $\left(\dot{x}_{r}, \dot{y}_{r}\right)$ plane. For tracking purposes, each pixel (i.e., PD) in the array is oversampled by a factor of $D_{r}^{2} / \delta^{2}$, where $\delta$ indicates the inter-spacing between the sub-pixels (i.e. sub-PDs). For instance, as illustrated in Fig.1, the PD is oversampled by a factor of 4, using a $2 \times 2$ sub-PD array. Such a PD is known as four-quad (F-Q) PD, commonly used for tracking purposes [19].

Consider Fig. 2 which illustrates the case of misaligned system. As shown, the transmitter axes are shifted in three directions by $\Delta_{x}, \Delta_{y}$, and $\Delta_{z}$. Also, the orientation of the transmitter axes are misaligned by three angles, namely, polar and azimuthal inclination angles $\left(\theta_{i n}, \phi_{i n}\right)$ and rotation angle $\phi_{r o}$. Notice that $\theta_{i n}, \phi_{i n}$ and $\phi_{r o}$ are the angles between axes $Z_{t}$ and $Z_{r}$, rotation around $Z_{t}$, and rotation around $Z_{r}$, respectively. This misalignment causes the basis of angle measurements at the transmitter and at the receiver to differ. Thus, though $\vec{e}_{m_{t}}$ is defined by angles $\left(\dot{\theta}_{m_{t}}, \dot{\phi}_{m_{t}}\right)$ relative to the transmitter axes as shown in Fig. 1, and it is written as

$$
\begin{gathered}
\vec{e}_{m_{t}}=\vec{x}_{m_{t}} \sin \left(\dot{\theta}_{m_{t}}\right) \cos \left(\dot{\phi}_{m_{t}}\right)+\vec{y}_{m_{t}} \sin \left(\dot{\theta}_{m_{t}}\right) \sin \left(\dot{\phi}_{m_{t}}\right) \\
+\vec{z}_{m_{t}} \cos \left(\dot{\theta}_{m_{t}}\right)
\end{gathered}
$$

$\vec{e}_{m_{t}}$ is also characterized by angles $\left(\theta_{m_{t}}, \phi_{m_{t}}\right)$ relative to the receiver axes as shown in Fig. 2, and can be equivalently written as

$$
\begin{gathered}
\vec{e}_{m_{t}}=\vec{x}_{r} \sin \left(\theta_{m_{t}}\right) \cos \left(\phi_{m_{t}}\right)+\vec{y}_{r} \sin \left(\theta_{m_{t}}\right) \sin \left(\phi_{m_{t}}\right) \\
+\vec{z}_{r} \cos \left(\theta_{m_{t}}\right)
\end{gathered}
$$


where $\left(\vec{x}_{t}, \vec{y}_{t}, \vec{z}_{t}\right)$ and $\left(\vec{x}_{r}, \vec{y}_{r}, \vec{z}_{r}\right)$ are the unit vectors relative to the axes of the transmitter and the receiver, respectively. Similarly, the received direction vector can be expressed as

$$
\vec{e}_{r}=\vec{x}_{r} \sin \left(\theta_{r}\right) \cos \left(\phi_{r}\right)+\vec{y}_{r} \sin \left(\theta_{r}\right) \sin \left(\phi_{r}\right)+\vec{z}_{r} \cos \left(\theta_{r}\right)
$$

\section{B. Characteristics of Angular MIMO Systems}

Though C-MIMO systems are similar to their A-MIMO counterparts in architecture, the collimating (i.e. convex) lens at the transmitter of A-MIMO greatly changes system characteristics. In particular, in a C-MIMO system, the magnification ${ }^{1}$ of the system will change with the range of the link making the received image larger when the transmitter is close and smaller with it is further away. The magnification for a C-MIMO architecture is given by [20]

$$
M_{C}=\frac{f_{r}}{L-f_{r}},
$$

where $L=L_{o}-\Delta_{z}$ is the total channel length. In contrast to C-MIMO, A-MIMO systems have a fixed magnification, given by [20]

$$
M_{A}=\frac{f_{r}}{f_{t}}
$$

This fixed-magnification means that the scale of the received image is independent of the link range, which is the first key advantage of A-MIMO systems.

A-MIMO systems can be viewed as transmitting data in the angular domain rather than in space, which is termed space-to-angle mapping [10]. As indicated in Fig. 1, the transmitter lens maps the beam of $m_{t}^{t h} \mathrm{LD}$ at position $\left(\dot{x}_{m_{t}}, \dot{y}_{m_{t}}\right)$ to associated polar and azimuthal transmitted angles $\left(\dot{\theta}_{m_{t}}, \dot{\phi}_{m_{t}}\right)$ as

$$
\begin{aligned}
& \dot{\theta}_{m_{t}}=\arctan \left(\sqrt{\dot{x}_{m_{t}}^{2}+\dot{y}_{m_{t}}^{2}} / f_{t}\right), \\
& \dot{\phi}_{m_{t}}= \begin{cases}\arccos \left(\dot{x}_{m_{t}} / \sqrt{\dot{x}_{m_{t}}^{2}+\dot{y}_{m_{t}}^{2}}\right), \quad \text { if } \quad \dot{y}_{m_{t}}<0, \\
\arccos \left(\dot{x}_{m_{t}} / \sqrt{\dot{x}_{m_{t}}^{2}+\dot{y}_{m_{t}}^{2}}\right)+\pi, \quad \text { if } \quad \dot{y}_{m_{t}} \geq 0 .\end{cases}
\end{aligned}
$$

\footnotetext{
${ }^{1}$ The magnification scale $M$ is given by $M:=\frac{I_{r}}{I_{t}}$, where $I_{r}$ and $I_{t}$ are the length of the received and transmitted images, respectively.
} 
TABLE I

A-MiMO Link PARAMETERS

$M_{t} \quad$ Number of LDs at the transmitter.

$D_{t} \quad$ Inter spacing between centres of LDs.

$d_{t} \quad$ Distance between LD array and the transmitter lens.

$f_{t} \quad$ Focal length of the transmitter lens.

$l_{t} \quad$ Diameter of the transmitter lens.

$\left(\dot{x}_{t}, \dot{y}_{t}\right) \quad$ Location in the plane of LD array relative

to the transmitter axis.

$\left(\dot{x}_{m_{t}}, \dot{y}_{m_{t}}\right) \quad$ Center of the $m_{t}{ }^{\text {th }}$ LD.

$g_{t}\left(\dot{x}_{t}, \dot{y}_{t}\right) \quad$ Transmitted image.

$\left(x_{t}, y_{t}\right) \quad$ Location of the ray on the transmit lens relative

to the link axis.

$\left(\dot{\theta}_{m_{t}}, \dot{\phi}_{m_{t}}\right) \quad$ Polar and azimuthal angles of the transmitted beam associated with $m_{t}{ }^{\text {th }} \mathrm{LD}$ relative to the transmitter axis.

$\left(\theta_{m_{t}}, \phi_{m_{t}}\right) \quad$ Polar and azimuthal angles of the transmitted beams associated with $m_{t}{ }^{\text {th }} \mathrm{LD}$ and relative to the link axis.

$M_{r} \quad$ Number of PDs at the receiver.

$D_{r} \quad$ Inter spacing between centres of PDs.

$d_{r} \quad$ Distance between the PD array and the receiver lens.

$f_{r} \quad$ Focal length of the receiver lens.

$l_{r} \quad$ Diameter of the receiver lens.

$\left(\dot{x}_{r}, \dot{y}_{r}\right) \quad$ Location in the plane of PD array relative to the link axis.

$\left(\dot{x}_{m_{r}}, \dot{y}_{m_{r}}\right) \quad$ Center of the $m_{r}{ }^{\text {th }}$ PD.

$\delta \quad$ Inter-spacing between sub-PDs in the PD.

$g_{r}\left(\dot{x}_{r}, \dot{y}_{r}\right) \quad$ Received image.

$\left(x_{r}, y_{r}\right) \quad$ Arrival positions of the rays in the receiver side relative to the link axis.

$\left(\theta_{r}, \phi_{r}\right) \quad$ Polar and azimuthal angles of the received beams relative to the link axis.

$L_{o} \quad$ Nominal length of the link.

$\left(\Delta_{x}, \Delta_{y}\right) \quad$ Off-axis between the transmitter axis and the link axis.

$\Delta_{z} \quad$ Variation in the channel length.

$\left(\theta_{i n}, \phi_{i n}\right) \quad$ Polar and azimuthal inclination angles of the transmitter relative to the link axis.

$\phi_{\text {ro }} \quad$ Rotation angle of the transmitter around the $Z_{t}$ axis. 
Notice that all LDs that are located at the same distance from the centre have the same polar transmitted angle but are distinguished by different azimuthal angles. Thus, the transmitter can be viewed as sending $M_{t}$ collimated beams to the receiver multiplexed spatially at $M_{t}$ unique polar and azimuthal angles.

The orientation misalignment causes launching angles $\left(\dot{\theta}_{m_{t}}, \dot{\phi}_{m_{t}}\right)$ and $\left(\theta_{m_{t}}, \phi_{m_{t}}\right)$ to differ. In this case, for given $\left(\theta_{i n}, \phi_{i n}, \phi_{r o}\right)$, angles $\left(\theta_{m_{t}}, \phi_{m_{t}}\right)$ are calculated from $\left(\dot{\theta}_{m_{t}}, \dot{\phi}_{m_{t}}\right)$ as follows: Let $\mathbf{e}_{m_{t}}$ be $(3 \times 1)$ vector, represented in $\left(X_{t}, Y_{t}, Z_{t}\right)$ as $\mathbf{e}_{m_{t}}=\left[\sin \left(\dot{\theta}_{m_{t}}\right) \cos \left(\dot{\phi}_{m_{t}}\right) ; \sin \left(\dot{\theta}_{m_{t}}\right) \sin \left(\dot{\phi}_{m_{t}}\right) ; \cos \left(\dot{\theta}_{m_{t}}\right)\right]$. Then, $\mathbf{e}_{m_{t}}$ is rotated around $\left(Y_{t}, X_{t}, Z_{t}\right)$ axes by three angles: $\theta_{y}=\arcsin \left(\cos \left(\phi_{i n}\right) \sin \left(\theta_{i n}\right)\right), \theta_{x}=$ $\arcsin \left(\sin \left(\phi_{i n}\right) \sin \left(\theta_{i n}\right) / \cos \left(\theta_{y}\right)\right)$ and $\theta_{z}=\phi_{r o}$ respectively. Thus, $\theta_{m_{t}}$ and $\phi_{m_{t}}$ are calculated by

$$
\begin{aligned}
\theta_{m_{t}} & =\arccos \left([0,0,1] \mathbf{R}_{z}\left(\theta_{z}\right) \mathbf{R}_{x}\left(\theta_{x}\right) \mathbf{R}_{y}\left(\theta_{y}\right) \mathbf{e}_{m_{t}}\right), \\
\phi_{m_{t}} & =\arcsin \left(\frac{[0,1,0]}{\sin \left(\theta_{m_{t}}\right)} \mathbf{R}_{z}\left(\theta_{z}\right) \mathbf{R}_{x}\left(\theta_{x}\right) \mathbf{R}_{y}\left(\theta_{y}\right) \mathbf{e}_{m_{t}}\right),
\end{aligned}
$$

where $\mathbf{R}_{z}\left(\theta_{z}\right), \mathbf{R}_{x}\left(\theta_{x}\right)$ and $\mathbf{R}_{y}\left(\theta_{y}\right)$ are $(3 \times 3)$ rotation matrices around $\left(Z_{t}, X_{t}, Y_{t}\right)$ axes respectively [21]. At the receiver, the angle-to-space mapping is done by the lens, which maps the received collimated beams at angle $\left(\theta_{r}, \phi_{r}\right)$ to the position $\left(\dot{x}_{r}, \dot{y}_{r}\right)$ on the PD plane, see Fig. 1. Using geometric optics and Eq. (6), the position $\left(\dot{x}_{r}, \dot{y}_{r}\right)$ is obtained by

$$
\begin{aligned}
& \dot{x}_{r}=f_{r} \tan \left(\theta_{r}\right) \cos \left(\phi_{r}\right), \\
& \dot{y}_{r}=\left\{\begin{array}{l}
\sqrt{\left(f_{r} \tan \left(\theta_{r}\right)\right)^{2}-\dot{x}_{r}^{2}}, \quad \text { if } \quad 0 \leq \phi_{r} \leq \pi, \\
-\sqrt{\left(f_{r} \tan \left(\theta_{r}\right)\right)^{2}-\dot{x}_{r}^{2}}, \quad \text { if } \quad \pi \leq \phi_{r} \leq 2 \pi .
\end{array}\right.
\end{aligned}
$$

Notice that, in case of perfect orientation (i.e., $\dot{\theta}_{m_{t}}=\theta_{m_{t}}, \dot{\phi}_{m_{t}}=\phi_{m_{t}}$ ), the space-to-angle mapping makes A-MIMO links resilient to shifting of the transmitter axes (i.e., $\Delta_{x}, \Delta_{y}, \Delta_{z}$ ). These affine translations of the transmitter relative to the receiver do not change the angle of reception and thus the received image is unaffected. This is the second key advantage of the A-MIMO over C-MIMO architecture. In practice, this advantage of A-MIMO links is limited by transceiver design constraints. Indeed, the performance of A-MIMO links is invariant to off-axis and channel length variation as long as $\Delta_{x}^{2}+\Delta_{y}^{2} \ll l_{r}^{2}$ and $L<L_{t h}$ are satisfied, respectively. The channel length threshold $L_{t h}$ depends on the size of the PD array and lens parameters and can be calculated by [16] 


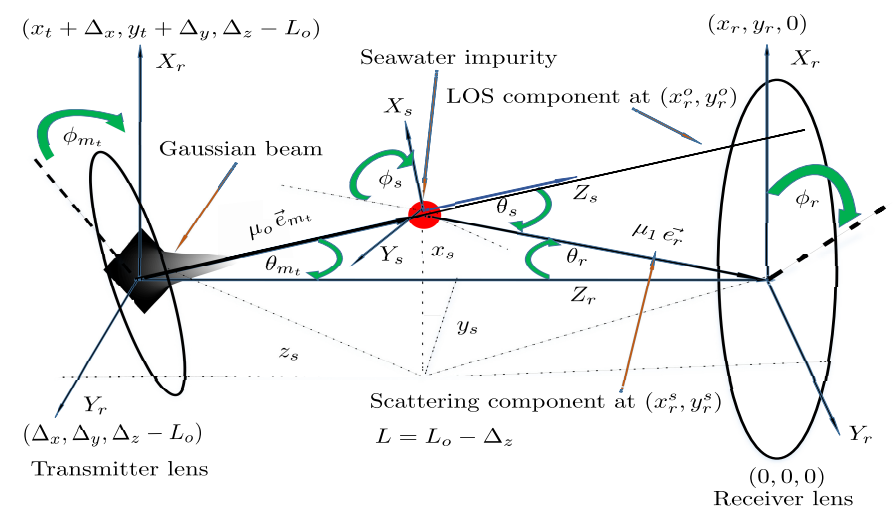

Fig. 3. Beam scattering model for an A-MIMO link, under single scattering assumption.

$$
L_{t h}=\frac{f_{r}\left(l_{r}+l_{t}\right)}{D_{r} \sqrt{M_{r}}} .
$$

In practice, the performance of A-MIMO links is degraded when $L>L_{t h}$. To illustrate, consider Fig. 1, if $L_{o}$ is increased too much, the red and green beams will be truncated and not detected. However, the central beam (in black) is received regardless of $L_{o}$. This process is termed window truncation [10] by which the received image is truncated by a circular aperture. In practice, $L_{t h}$ can be extended by increasing the transceiver size (e.g. $f_{r}, l_{r}$ and $l_{t}$ ) as given in Eq. (9) [16]. Thus, given this limitation of window truncation for long ranges, A-MIMO systems are best suited to short-range applications.

In the case of orientation misalignment, i.e. $\dot{\theta}_{m_{t}} \neq \theta_{m_{t}}, \dot{\phi}_{m_{t}} \neq \phi_{m_{t}}$, the angles of the received ray will be shifted relative to the receiver optical axis and thus the received image will also be shifted. For example, the inclination by angles $\left(\theta_{i n}, \phi_{i n}\right)$ and rotation by angle $\phi_{\text {ro }}$ lead to shifting and rotating of the received image, as shown in Fig. 2. An interesting point is that the geometric optics relationships inherent to A-MIMO can be used to estimate the misalignment parameters from the received image, as we will show in Section V.

\section{UNDERWATER A-MIMO LINK MODELling}

In this section, a mathematical model is derived for an underwater A-MIMO link, taking into account link misalignment, scattering, beam attenuation, and background noise. For beam attenuation in seawater, we consider beam attenuation and single scattering, which is valid for short-range, low-turbidity UWOC [22]-[24]. In the case of short-range communications 
considered here, it is reasonable to ignore the impact of turbulence since the variation in the refractive index of the seawater is negligible. Also, due to the use of relatively large receive apertures at the receiving side, aperture averaging will further reduce the impact of any turbulence [25]. In the following analysis, the AoA distribution of the received signal is derived first. Then, the probability density function (PDF) of PoA is obtained from the AoA PDF using the random variable transformation (RVT) theory. Using the latter, the channel matrix and the received image are calculated.

\section{A. AoA Distribution}

Figure 3 illustrates transmitter with displacement and disorientation with respect to the receiver. Consider that the optical beam from the $m_{t}{ }^{\text {th }}$ LD has the optical power $P_{o}$ with a Gaussian beam profile [26]

$$
I_{m_{t}}\left(x_{t}, y_{t}\right)=I_{o} \exp \left(\frac{-2\left(\left(x_{t}-x_{m_{t}}\right)^{2}+\left(y_{t}-y_{m_{t}}\right)^{2}\right)}{w_{o}^{2}}\right)
$$

where $w_{o}$ and $\left(x_{m_{t}}, y_{m_{t}}\right)$ are the beam waist and beam centre coordinates, respectively. Moreover, the radius of the beam width on the transmitter lens is $w=w_{o} \sqrt{\left(1+\lambda d_{t} /\left(\pi w_{o}^{2}\right)\right)^{2}}$ with $\lambda$ being the wavelength. In order to simplify the analysis, we assume that $w_{o} \ll D_{t}$, which allows approximating the $m_{t}^{\text {th }} \mathrm{LD}$ as a point-source at position $\left(\dot{x}_{m_{t}}, \dot{y}_{m_{t}}\right)$.

Seawater impurities in underwater channels cause absorption and scattering of the transmitted optical beam. The extinction ratio $c=a+b$ and albedo coefficient $W=b / c$ quantify the total power loss and single scattering, respectively, where $a$ and $b$ are the absorption and scattering coefficients, respectively [27]. Under a single scattering assumption, a transmitted ray is scattered at most once during its propagation between the transmitter and the receiver. More precisely, a ray sent in the direction $\vec{e}_{m_{t}}$, after propagating over a given distance, is attenuated and randomly scattered to a new direction $\vec{e}_{r}$, which can be divided into line-of-sight (LOS) and scattered components, as shown in Fig. 3. For the LOS component, the amplitude of the optical ray is attenuated according to the Beer-Lambert law, however, its direction is maintained (i.e., $\vec{e}_{m_{t}}=\vec{e}_{r}$ ) arriving at position $\left(x_{r}^{o}, y_{r}^{o}\right)$ as shown in the figure. For the scattered component, scattering leads to the likelihood of loss by a factor $b / c$. The likelihood of ray-impurity interaction ${ }^{2}$. after

\footnotetext{
${ }^{2}$ The ray-impurity interaction is either an absorption or scattering event. The absorption event results when ray losses all energy (i.e. ray termination), while the scattering event occurs when the ray changes its direction (i.e. ray deflection) [28]
} 
propagating over a distance $\mu_{0}$, is modelled by an exponential PDF [22], [28]

$$
p_{\mu}\left(\mu_{0}\right)=c \exp \left(-c \mu_{0}\right)
$$

Upon scattering, a incindent ray arriving from the direction $\vec{e}_{m_{t}}$ will have its direction $\vec{e}_{r}$ changed randomly according to polar and azimuthal scattering angles $\left(\theta_{s}, \phi_{s}\right)$. A common probabilistic model for $\theta_{s}$ is given by [29]

$$
p_{\theta_{s}}\left(\theta_{s}\right)=\frac{1-g^{2}}{4 \pi\left(1+g^{2}-2 g \cos \left(\theta_{s}\right)\right)^{(3 / 2)}}, \quad g=\overline{\cos \left(\theta_{s}\right)}
$$

where, the average cosine $g$ depends on the seawater type. Also, azimuthal scattering angle is typically described by the uniform PDF [30]

$$
p_{\phi_{s}}\left(\phi_{s}\right)=\frac{1}{2 \pi}
$$

For the given scattering angle $\left(\theta_{s}, \phi_{s}\right), \vec{e}_{r}$ is calculated from $\vec{e}_{m_{t}}$ using Eq. (7), by replacing the orientation misalignment angles $\left(\theta_{i n}, \phi_{i n}, \phi_{r o}\right)$ by $\left(\theta_{s}, \phi_{s}, 0\right)$ in the definitions of $\theta_{x}, \theta_{y}$, and $\theta_{z}$. After being scattered, the ray travels a distance $\mu_{1}$ in the direction $\vec{e}_{r}$ before arriving at the receiver at the position $\left(x_{r}^{s}, y_{r}^{s}\right)$, as shown in Fig. 3. The ray is effectively detected if PoA is located within the receiver lens aperture. However, the ray may contribute to spatial inter-channel interference (i.e. cross-talk) depending on which PD it is incident. Mathematically, a ray arriving at position $\left(x_{r}, y_{r}\right)$ is effectively received provided that $\left(x_{r}^{2}+y_{r}^{2}\right) \leq l_{r}^{2} / 4$. For the $m_{t}{ }^{\text {th }} \mathrm{LD}$, the corresponding geometric loss, denoted by $G_{m_{t}}$, which takes the window truncation into account, is given by

$$
G_{m_{t}}\left(x_{r}, y_{r}\right)= \begin{cases}1, & \text { if } \quad\left(x_{r}^{2}+y_{r}^{2}\right) \leq l_{r}^{2} / 4 \\ 0, & \text { otherwise }\end{cases}
$$

In short-range, low-albedo waters (e.g., $W=0.053$ in case of pure seawaters) the impact of scattering is small [27]. Thus, the AoA PDF of the received power can be well approximated by a linear combination of LOS components from each LD. For an $\left(\sqrt{M_{t}} \times \sqrt{M_{t}}\right)$ LD array, the AoA distribution of the received signal can be approximated as 


$$
\begin{aligned}
& P_{\theta_{r}, \phi_{r}}\left(\theta_{r}, \phi_{r}\right) \approx \sum_{m_{t}=1}^{M_{t}} \exp \left(\frac{-L c}{\cos \left(\theta_{m_{t}}\right)}\right) \int_{x_{t_{n}}}^{x_{t_{p}}} \int_{y_{t_{n}}}^{y_{t_{p}}} I_{m_{t}}\left(x_{t}, y_{t}\right) \\
& \times G_{m_{t}}\left(x_{r}^{o}, y_{r}^{o}\right) d y_{t} d x_{t} \delta\left(\theta_{r}-\theta_{m_{t}}, \phi_{r}-\phi_{m_{t}}\right) .
\end{aligned}
$$

In Eq. (15), the exponential term is the path loss according to the Beer-Lambert law [30]. The limits of the integrals are defined as: $x_{t_{n}}=-l_{t} / 2+\Delta_{x}, x_{t_{p}}=l_{t} / 2+\Delta_{x}, y_{t_{n}}=-l_{t} / 2+\Delta_{y}$ and $y_{t_{p}}=l_{t} / 2+\Delta_{y}$. The arrival position of the LOS ray is shown in Fig. 3, which it is given by $x_{r}^{o}=x_{t}+\Delta_{x}+L \tan \left(\theta_{t}\right) \cos \left(\phi_{t}\right)$, and $y_{r}^{o}=y_{t}+\Delta_{y}+L \tan \left(\theta_{t}\right) \sin \left(\phi_{t}\right)$. Define $\delta\left(\theta_{r}-\theta_{m_{t}}, \phi_{r}-\phi_{m_{t}}\right)$ as the Dirac-delta function, taking its non-zero value at the associated launching angles. Note that for the case of relatively high-albedo seawaters, multiple scattering can become significant, especially for relatively long-range links [22]. In the following analysis, we consider only the LOS and single scattering components that dominate short-range links, which allows a tractable mathematical analysis of the link misalignment.

In order to calculate the single scattering component, for a transmitted ray in direction $\vec{e}_{m_{t}}$ and scattered at point $\left(x_{s}, y_{s}, z_{s}\right)$ with scattering angle $\theta_{s}$ (see Fig. 3), we have [12]

$$
\begin{array}{rr}
x_{s}=\mu_{0} \sin \left(\theta_{m_{t}}\right) \cos \left(\phi_{m_{t}}\right), \quad y_{s}=\mu_{0} \sin \left(\theta_{m_{t}}\right) \sin \left(\phi_{m_{t}}\right), \\
z_{s}=-\mu_{0} \cos \left(\theta_{m_{t}}\right), & \theta_{s}=\arccos \left(\vec{e}_{m_{t}} \cdot \vec{e}_{r}\right) .
\end{array}
$$

The arrival position of the scattered ray $\left(x_{r}^{s}, y_{r}^{s}, 0\right)$ in the receiving side is [12]

$$
\begin{aligned}
& x_{r}^{s}=x_{t}+\Delta_{x}+x_{s}+\mu_{1} \sin \left(\theta_{r}\right) \cos \left(\phi_{r}\right), \\
& y_{r}^{s}=y_{t}+\Delta_{y}+y_{s}+\mu_{1} \sin \left(\theta_{r}\right) \sin \left(\phi_{r}\right), \\
& 0=L_{o}-\Delta_{z}+z_{s}-\mu_{1} \cos \left(\theta_{r}\right) .
\end{aligned}
$$

Using Eqs. (10)-(17), the AoA distribution of LOS and scattering components is derived by using a similar approach as in [22]. Accordingly, the AoA distribution of received signal from the $m_{t}^{\text {th }} \mathrm{LD}$ is given as 


$$
\begin{aligned}
& P_{\theta_{r}, \phi_{r}}\left(\theta_{r}, \phi_{r} \mid \theta_{m_{t}}, \phi_{m_{t}}\right)=\int_{x_{t_{n}}}^{x_{t_{p}}} \int_{y_{t_{n}}}^{y_{t_{p}}} I_{m_{t}}\left(x_{t}, y_{t}\right)\left(\frac{b}{4 \pi} \times\right. \\
& p_{\phi_{s}}\left(\phi_{s}\right) p_{\theta_{s}}\left(\arccos \left(\vec{e}_{m_{t}} \cdot \vec{e}_{r}\right)\right) \sin \left(\arccos \left(\vec{e}_{m_{t}} \cdot \vec{e}_{r}\right)\right) \\
& \times \int_{0}^{l_{\mu_{o}}} \exp \left(-c \mu_{0}\right) \exp \left(-c \mu_{1}\right) G_{m_{t}}\left(x_{r}^{s}, y_{r}^{s}\right) d \mu_{0} \\
& \left.+\exp \left(\frac{-c L}{\cos \left(\theta_{m_{t}}\right)}\right) \times G_{m_{t}}\left(x_{r}^{o}, y_{r}^{o}\right) \delta\left(\theta_{r}-\theta_{m_{t}}, \phi_{r}-\phi_{m_{t}}\right)\right)
\end{aligned}
$$

$d y_{t} d x_{t}$

where $l_{\mu_{o}}=L / \cos \left(\theta_{m_{t}}\right)$ and $P_{\theta_{r}, \phi_{r}}\left(\theta_{r}, \phi_{r} \mid \theta_{m_{t}}, \phi_{m_{t}}\right)$ has units of $\mathrm{W} / \mathrm{rad}^{2}$. The first and the second terms in Eq. (18) refer to the scattered and LOS components, respectively.

A simpler AoA expression can be derived from Eq. (18) assuming that the beam spot on the transmitter lens is very small, i.e., $w<<l_{t}$, allowing the LD to be well approximated as a single-ray source. For the central LD under perfect beam alignment (i.e., $x_{t}=y_{t}=0$ and $\Delta_{x}=\Delta_{y}=0$ ), Eq. (18) can be simplified as

$$
\begin{aligned}
& P_{\theta_{r}, \phi_{r}}\left(\theta_{r}, \phi_{r} \mid \theta_{m_{t}}, \phi_{m_{t}}\right)=\frac{P_{o} b\left(1-g^{2}\right) \sin \left(\arccos \left(\vec{e}_{m_{t}} \cdot \vec{e}_{r}\right)\right)}{8 \pi^{2}\left(1+g^{2}-2 g\left(\vec{e}_{m_{t}} \cdot \vec{e}_{r}\right)\right)^{3 / 2}} \\
& \times \frac{\exp \left(-c L \sec \left(\theta_{r}\right)\right)\left(\exp \left(\nu f_{2}\left(\theta_{r}, \phi_{r}\right)\right)-\exp \left(\nu f_{1}\left(\theta_{r}, \phi_{r}\right)\right)\right)}{c\left(\cos \left(\theta_{m_{t}}\right) \sec \left(\theta_{r}\right)-1\right)} \\
& +P_{o} \exp \left(\frac{-c L}{\cos \left(\theta_{m_{t}}\right)}\right) G_{m_{t}}\left(x_{r}^{o}, y_{r}^{o}\right) \delta\left(\theta_{r}-\theta_{m_{t}}, \phi_{r}-\phi_{m_{t}}\right),
\end{aligned}
$$

where $\nu=c\left(\cos \left(\theta_{m_{t}}\right) \sec \left(\theta_{r}\right)-1\right)$.

Integrating Eq. (19) over $\phi_{r}$ gives the polar AoA distribution as

$$
\begin{aligned}
& P_{\theta_{r}}\left(\theta_{r} \mid \theta_{m_{t}}\right)=P_{o} \exp \left(\frac{-c L}{\cos \left(\theta_{m_{t}}\right)}\right) G_{m_{t}}\left(x_{r}^{o}, y_{r}^{o}\right) \delta\left(\theta_{r}-\theta_{m_{t}}\right) \\
& +\frac{P_{o} b\left(1-g^{2}\right) \sin \left(\arccos \left(\vec{e}_{m_{t}} \cdot \vec{e}_{r}\right)\right) \exp \left(-c L \sec \left(\theta_{r}\right)\right)}{8 \pi^{2} c\left(1+g^{2}-2 g\left(\vec{e}_{m_{t}} \cdot \vec{e}_{r}\right)\right)^{3 / 2}\left(\cos \left(\theta_{m_{t}}\right) \sec \left(\theta_{r}\right)-1\right)} \\
& \times \int_{0}^{2 \pi}\left(\exp \left(\nu f_{2}\left(\theta_{r}, \phi_{r}\right)\right)-\exp \left(\nu f_{1}\left(\theta_{r}, \phi_{r}\right)\right)\right) d \phi_{r} .
\end{aligned}
$$

In particular, for zero launching angle (i.e. $\theta_{m_{t}}=0$ ), Eq. (20) is simplified as 


$$
\begin{aligned}
& P_{\theta_{r}}\left(\theta_{r} \mid \theta_{m_{t}}=0\right)=\frac{P_{o} b\left(1-g^{2}\right) \sin \left(\theta_{r}\right) \exp \left(-c L \sec \left(\theta_{r}\right)\right)}{c\left(\sec \left(\theta_{r}\right)-1\right)\left(1+g^{2}-2 g \cos \left(\theta_{r}\right)\right)^{3 / 2}} \\
& \frac{1}{4 \pi}\left(\exp \left(c \frac{f_{2}\left(\theta_{r}\right)}{\left(\sec \left(\theta_{r}\right)-1\right)^{-1}}\right)-\exp \left(c \frac{f_{1}\left(\theta_{r}\right)}{\left(\sec \left(\theta_{r}\right)-1\right)^{-1}}\right)\right) \\
& +P_{o} \exp \left(\frac{-c L}{\cos \left(\theta_{m_{t}}\right)}\right) G_{m_{t}}\left(x_{r}^{o}, y_{r}^{o}\right) \delta\left(\theta_{r}-\theta_{m_{t}}, \phi_{r}-\phi_{m_{t}}\right) .
\end{aligned}
$$

Similarly, the azimuthal AoA distribution can be obtained from Eq. (19) as

$$
\begin{aligned}
& P_{\phi_{r}}\left(\phi_{r} \mid \theta_{m_{t}}, \phi_{m_{t}}\right)= \\
& P_{o}\left(\exp \left(\frac{-c L}{\cos \left(\theta_{m_{t}}\right)}\right) G_{m_{t}}\left(x_{r}^{o}, y_{r}^{o}\right) \delta\left(\phi_{r}-\phi_{m_{t}}\right)+\right. \\
& \frac{b\left(1-g^{2}\right)}{8 \pi^{2} c} \int_{0}^{\pi / 2} \frac{\sin \left(\arccos \left(\vec{e}_{m_{t}} \cdot \vec{e}_{r}\right)\right) \exp \left(-c L \sec \left(\theta_{r}\right)\right)}{\left(1+g^{2}-2 g\left(\vec{e}_{m_{t}} \cdot \vec{e}_{r}\right)\right)^{3 / 2}} \\
& \left.\times \frac{\left(\exp \left(\nu f_{2}\left(\theta_{r}, \phi_{r}\right)\right)-\exp \left(\nu f_{1}\left(\theta_{r}, \phi_{r}\right)\right)\right)}{\left(\cos \left(\theta_{m_{t}}\right) \sec \left(\theta_{r}\right)-1\right)} d \theta_{r}\right) .
\end{aligned}
$$

Equation (22) can be approximated in a closed-form using Eq. (21) for $\theta_{m_{t}}=0$ as

$$
\begin{aligned}
& P_{\phi_{r}}\left(\phi_{r} \mid \theta_{m_{t}}=0\right) \approx \frac{P_{o} b \exp (-c L)}{11.63 \pi c} \times \\
& \left(1.45 \times 10^{3}-\sum_{i=1}^{2} \xi_{i}\left(\Gamma\left(\gamma_{i}, \alpha_{i}\right)-\Gamma\left(\gamma_{i}, \beta_{i}\right)\right)\right. \\
& +\frac{2258.5}{\sqrt{c L}}\left(\operatorname{erf}\left(\sqrt{\frac{c L}{2}} \operatorname{arccot}\left(\frac{c L}{l_{r}}\right)\right)-\operatorname{erf}\left(\frac{\sqrt{c L}}{81.03}\right)\right) \\
& \left.-\frac{7208}{c l_{r}}\left(\exp \left(\frac{-c l_{r}}{19.1}\right)-\exp \left(\frac{-c l_{r}}{4} \operatorname{arccot}\left(\frac{2 L}{l_{r}}\right)\right)\right)\right),
\end{aligned}
$$

where, the incomplete-gamma function $\Gamma(\gamma, \alpha)$ and the error function $\operatorname{erf}(u)$ are defined as: $\Gamma(\gamma, \alpha)=\int_{\alpha}^{\infty} t^{\gamma-1} \exp (-t) d t$ and $\operatorname{erf}(u)=\frac{2}{\sqrt{\pi}} \int_{0}^{u} \exp \left(-t^{2}\right) d t$. Also, the coefficients of incomplete-gamma functions are defend as: $\xi_{1}=-603.47(c L)^{0.26}, \xi_{2}=76.07\left(c l_{r}\right)^{0.52}, \gamma_{1}=$ $-0.26, \gamma_{2}=-0.52, \alpha_{1}=c l / 2\left(\cot ^{-1}\left(2 L / l_{r}\right)\right)^{2}, \alpha_{2}=c l_{r} / 19.12, \beta_{1}=c L / 114.6$ and $\beta_{2}=$ $c l_{r} / 4 \cot ^{-1}\left(2 L / l_{r}\right)$.

Notice that, though Eq. (18) is the exact distribution of the AOA, Eqs. (19)-(23) provide simpler closed-forms, which are more convenient to use. Detailed proofs of Eqs. (19), (21) and (23) are provided in Appendix A. In Section VI the accuracy of Eqs. (19-23) is verified using MCNRT simulations. 


\section{B. PoA Distribution}

Recall that for A-MIMO systems, the receiver lens maps the AoA to a PoA on the focal plane of the imaging receiver and onto the PD array. In order to simplify the derivation of PoA, the field of view (FOV) of the PDs is assumed to be full angle ${ }^{3}$, i.e., $180^{\circ}$. Thus, the received power of PoA distribution from the $m_{t}^{\text {th }}$ LD is derived from Eqs. (18) and (8) as

$$
P_{\dot{x}_{r}, \dot{y}_{r}}\left(\dot{x}_{r}, \dot{y}_{r} \mid \theta_{m_{t}}, \phi_{m_{t}}\right)=\frac{f_{r} P_{\theta_{r}, \phi_{r}}\left(\dot{x}_{r}, \dot{y}_{r} \mid \theta_{m_{t}}, \phi_{m_{t}}\right)}{\left(f_{r}^{2}+\dot{x}_{r}^{2}+\dot{y}_{r}^{2}\right) \sqrt{\left(\dot{x}_{r}^{2}+\dot{y}_{r}^{2}\right)}}
$$

where the distribution is in units of $\mathrm{W} / \mathrm{m}^{2}$. Also, the focal plane of the imaging receiver is defined by the square region $-\sqrt{M_{r}} D_{r} / 2 \leq\left\{\dot{x}_{r}, \dot{y}_{r}\right\} \leq \sqrt{M_{r}} D_{r} / 2$.

Using Eq. (24), the channel gain between the $m_{t}^{\text {th }}$ LD and the $m_{r}^{\text {th }}$ PD can be calculated as

$$
h_{m_{r}, m_{t}}(\boldsymbol{\Delta})=\frac{1}{P_{o}} \int_{x_{r_{n}}}^{x_{r_{p}}} \int_{y_{r_{n}}}^{y_{r_{p}}} P_{\dot{x}_{r}, \dot{y}_{r}}\left(\dot{x}_{r}, \dot{y}_{r} \mid \theta_{m_{t}}, \phi_{m_{t}}\right) d \dot{y}_{r} d \dot{x}_{r}
$$

where $\boldsymbol{\Delta}:=\left[\theta_{\text {in }} ; \phi_{i n} ; \phi_{\text {ro }} ; \Delta_{x} ; \Delta_{y} ; \Delta_{z}\right]$ is the $(6 \times 1)$ misalignment vector, and the limits of the integral are defined as $x_{r_{n}}=\dot{x}_{m_{r}}-D_{r} / 2, x_{r_{p}}=\dot{x}_{m_{r}}+D_{r} / 2, y_{r_{n}}=\dot{y}_{m_{r}}-D_{r} / 2$ and $y_{r_{p}}=\dot{y}_{m_{r}}+D_{r} / 2$. For an $\left(M_{t} \times M_{r}\right)$ A-MIMO system, the channel gains can be arranged in a channel matrix $\mathbf{H}$ defined as

$$
\mathbf{H}(\boldsymbol{\Delta})=\left[\begin{array}{cccc}
h_{11} & h_{12} & \ldots & h_{1, M_{t}} \\
h_{21} & h_{22} & \ldots & h_{2, M_{t}} \\
\vdots & \vdots & h_{m_{r}, m_{t}} & \vdots \\
h_{M_{r}, 1} & h_{M_{r}, 2} & \ldots & h_{M_{r}, M_{t}}
\end{array}\right] .
$$

The correlation of the channel matrix depends on the transceiver parameters (i.e., $D_{t}, l_{t}, D_{r}$ and $l_{r}$ ) and the channel conditions (i.e., $a, b$ and $L$ ). For example, increasing the inter-spacing between LDs/PDs leads to a reduced spatial inter-channel interference, which reduces the channel correlation. As well, increasing the channel length or seawater turbidity leads to an increase in spatial inter-channel interference, which increases the channel correlation.

Due to displacement and orientation misalignments the received image is a truncated, shifted and rotated version of the image at the transmitter focal plane. Additionally, absorption and

\footnotetext{
${ }^{3}$ The full FOV maximizes the channel gain, however it is not ideal in case of high background noise. In the presented work, in order to reduce the effects of the background noise, an optical filter is implemented with the receiver lens.
} 
scattering due to seawater will result in an attenuated and blurred received image. For the $\left(\sqrt{M_{t}} \times \sqrt{M_{t}}\right)$ LD array, using Eq. (24), the received image is a summation of $M_{t}$ received PoA distributions and noise $n\left(\dot{x}_{r}, \dot{y}_{r}\right)$

$$
g_{r}\left(\dot{x}_{r}, \dot{y}_{r}\right)=\sum_{m_{t}=1}^{M_{t}} P_{\dot{x}_{r}, \dot{y}_{r}}\left(\dot{x}_{r}, \dot{y}_{r} \mid \theta_{m_{t}}, \phi_{m_{t}}\right)+n\left(\dot{x}_{r}, \dot{y}_{r}\right) .
$$

The noise term $n\left(\dot{x}_{r}, \dot{y}_{r}\right)$ is the summation of the internal and external noises, denoted by $n_{i n}\left(\dot{x}_{r}, \dot{y}_{r}\right)$ and $n_{e x}\left(\dot{x}_{r}, \dot{y}_{r}\right)$, respectively. The former includes thermal, shot and dark noises, whereas the latter refers to the background noise [31]. For the background noise, we consider the worst-case analysis proposed in [31, Eq. (9)], corresponding to a vertical link with unobstructed sunlight from the zenith. Using Eq. (6), we have

$$
n_{e x}\left(\dot{x}_{r}, \dot{y}_{r}\right)=\frac{\pi l_{r}^{2} \lambda_{\Delta} E_{s}}{4 \exp \left(K_{d} L_{h}\right)} \cos \left(\arctan \left(\frac{\sqrt{\dot{x}_{r}^{2}+\dot{y}_{r}^{2}}}{f_{r}}\right)\right),
$$

where, $E_{s}$ is the solar intensity and $L_{h}$ is the depth of the receiver. The light diffusion coefficient, $K_{d}$, is related to water type and the water turbidity $W$. As well, $\lambda_{\Delta}$ is the optical bandwidth of the passband receiver optical filter, centred at the wavelength of the LD. The use of an optical filter is very effective in order to mitigate background noise especially when the orientation of the receiver is upwards [31].

For simplicity, assume that the receiver noise is signal independent, and use an additive white Gaussian noise (AWGN) model for $n\left(\dot{x}_{r}, \dot{y}_{r}\right)$ [32].

\section{Channel Capacity and Misalignment Models}

Considering the AWGN model, the $\left(M_{r} \times 1\right)$ vector $\mathbf{y}$ of the receiver photo-currents, is given by:

$$
\mathbf{y}=\Re \mathbf{H}(\boldsymbol{\Delta}) \mathbf{x}+\mathbf{n}
$$

where, $\Re$ is the PD responsitivity, $\mathrm{x}$ is the $\left(M_{t} \times 1\right)$ transmitted optical power vector and $\Delta$ is defined in Eq. (25). Also, $\mathbf{n}=\left[n_{1} ; n_{2} ; \ldots ; n_{m_{r}} ; \ldots ; n_{M_{r}}\right]$ is the $\left(M_{r} \times 1\right)$ noise vector where the noise element $n_{m_{r}}$ is quantified as

$$
n_{m_{r}}=\int_{x_{r_{n}}}^{x_{r_{p}}} \int_{y_{r_{n}}}^{y_{r_{p}}}\left(n_{e x}\left(\dot{x}_{r}, \dot{y}_{r}\right)+n_{i n}\left(\dot{x}_{r}, \dot{y}_{r}\right)\right) d \dot{y}_{r} d \dot{x}_{r}
$$




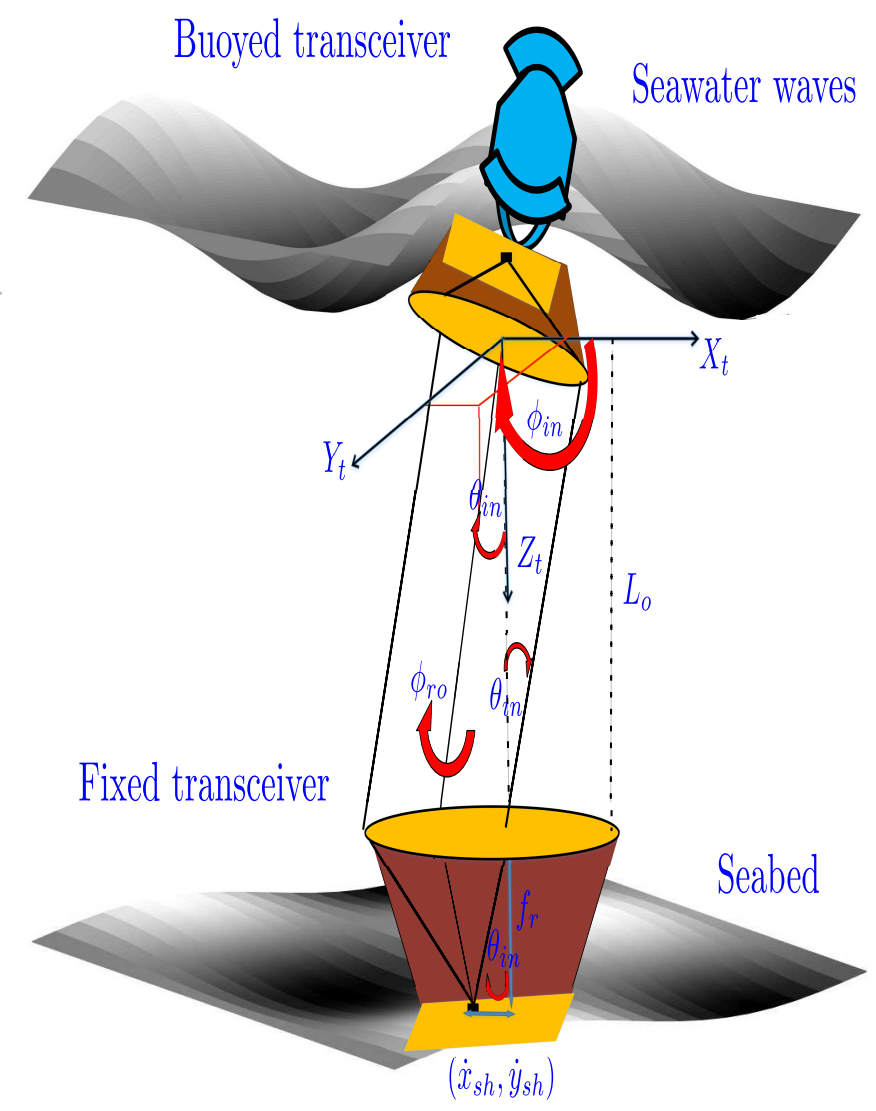

Fig. 4. Buoyed-to-Fixed (B2F) communication model: Inclination and rotation angles $\left(\theta_{i n}, \phi_{i n}, \phi_{r o}\right)$ due to waves of seawaters (i.e. surface slop).

where the integration is taken over each PD as in Eq. (25). In the case where the channel state information is not available at the transmitter (e.g., unknown seawater parameters), the total transmitted average optical power $P_{t}$ is divided equally among all LDs (i.e., $P_{o}=P_{t} / M_{t}$ ). For an imaging receiver with independent identical Gaussian noise elements with zero mean and variance $\sigma_{n}^{2}$ (i.e., $n_{m_{r}} \sim \mathcal{N}\left(0, \sigma_{n}^{2}\right)$ ), the link capacity in bits/channel-use is obtained as [33]

$$
C(\boldsymbol{\Delta})=\frac{1}{2} \log _{2}\left(\operatorname{det}\left(\mathbf{I}+\left(\frac{\Re P_{o}}{\sigma_{n}}\right)^{2} \mathbf{H}(\boldsymbol{\Delta}) \mathbf{H}(\boldsymbol{\Delta})^{T}\right)\right),
$$

where $\mathbf{I}$ is the identity matrix and $(.)^{T}$ denotes matrix transposition.

Equation (31) implicitly assumes DCO-OFDM (DC-biased optical orthogonal frequency division multiplexing) signalling, which is a spectrally efficient technique to overcome the bandwidth limitation of the optoelectronic components [34], [35]. In the following, the link capacity is evaluated for $\mathrm{B} 2 \mathrm{~F}$ and $\mathrm{M} 2 \mathrm{~F}$ communication scenarios. 


\section{A. B2F Communication Model}

Figure 4 illustrates a typical B2F communication scenario where one transceiver is tied to a buoyed node on the sea surface and the other one is fixed on the seabed. Such links are commonly used in low depth seawaters, e.g., for high-speed communication with underwater sensor networks [36]. Here, surface waves disrupt the orientation of the buoyed node, causing random inclination and rotation angles. For simplicity, in this scenario it is assumed that the changes in $X_{t}, Y_{t}$ and $Z_{t}$ are negligible, that is, we consider $\Delta_{x}=\Delta_{y}=\Delta_{z}=0$.

Cox et al. developed in [37] a model for seawater waves based on an isotropic Gaussian distribution. According to this model, only $\theta_{i n}$ and $\phi_{i n}$ orientation misalignments are considered and described by a joint PDF. Here, we extend the Cox model to include an independent and uniform rotation misalignment of $\phi_{\text {ro }}$ resulting in the following PDF,

$$
\begin{aligned}
p_{\theta_{i n}, \phi_{i n}, \phi_{r o}}\left(\theta_{i n}, \phi_{i n}, \phi_{r o}\right)=\frac{\tan \left(\theta_{i n}\right) \sec ^{2}\left(\theta_{i n}\right)}{(2 \pi)^{2} \sigma_{i n}^{2}} \\
\times \exp \left(-\frac{\tan ^{2}\left(\theta_{i n}\right)}{2 \sigma_{i n}^{2}}\right)
\end{aligned}
$$

where $\sigma_{i n}^{2}$ is the variance of the slope of the sea surface (i.e., sea waves), which increases linearly with wind speed $U$ in $\mathrm{m} / \mathrm{sec}$ through the relationship $\sigma_{i n}^{2}=0.003+0.00512 U$ for $0 \mathrm{~m} / \mathrm{sec} \leq U \geq 16 \mathrm{~m} / \mathrm{sec}$ [37]. In order to simplify capacity calculations two scenarios are considered separately, denoted by $S 1$ and $S 2$, assuming $\phi_{i n}=0$ and $\phi_{r o}=0$, respectively. Using Eqs. (31) and (32), the average capacity of A-MIMO and C-MIMO links can be obtained as

$$
\bar{C}(U)=\int_{0}^{2 \pi} \int_{0}^{\pi / 2} C(\boldsymbol{\Delta}) p_{\theta_{i n}, \phi_{\chi}}\left(\theta_{i n}, \phi_{\chi}\right) d \theta_{i n} d \phi_{\chi}
$$

where $\phi_{\chi}$ is equal to $\phi_{\text {ro }}$ and $\phi_{\text {in }}$ for scenarios $S 1$ and $S 2$, respectively.

\section{B. M2F Communication Model}

Figure 5 illustrates a typical M2F communication scenario, where one of the transceivers is fixed on an AUV and the other one on the seabed. Such M2F links are commonly used for linking AUVs with an underwater sensor network located on the seabed, e.g., for underwater mining or for monitoring gas/oil pipelines [38]. Here, sea currents can disrupt the alignment 


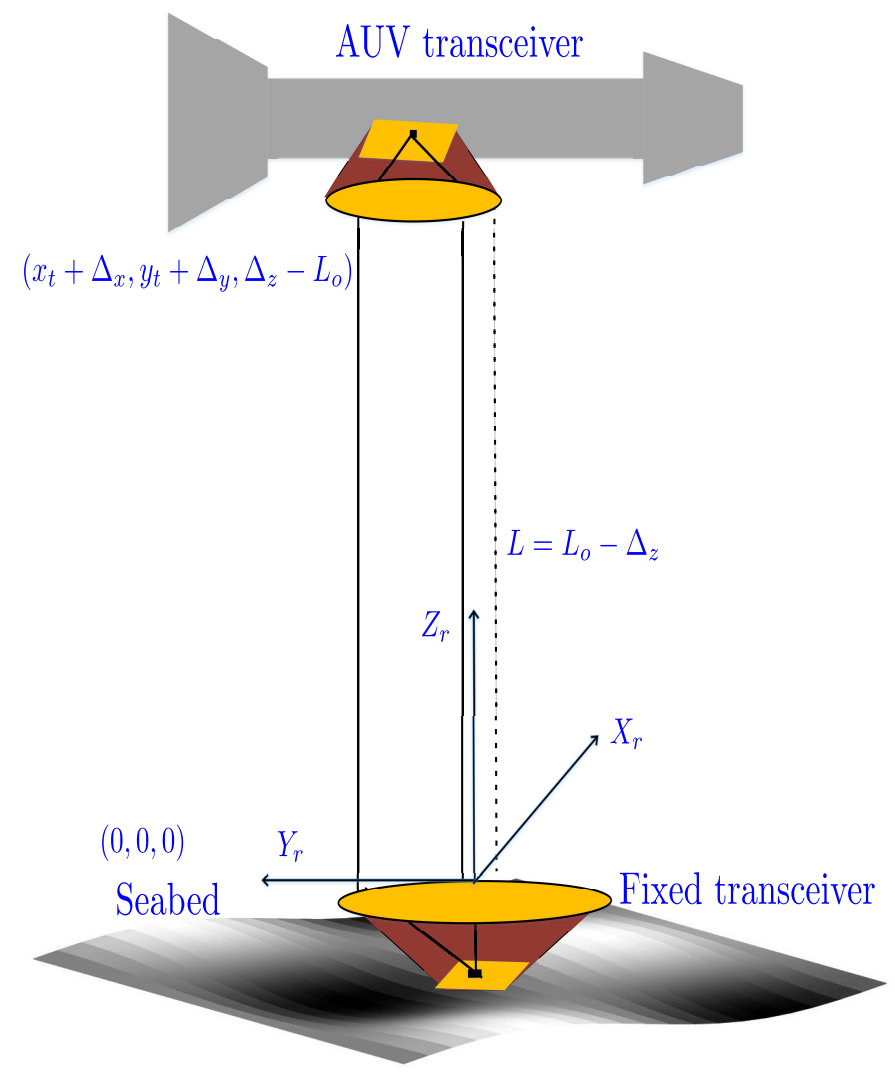

Fig. 5. M2F communication model showing off-axis transmission with displacement $\left(\Delta_{x}, \Delta_{y}\right)$ and channel length variation $\left(\Delta_{z}\right)$.

between the AUV and the fixed sensor node, causing the AUV to be displaced in the three axes as shown in Fig. 5. For simplicity, in this scenario perfect angular orientation between the two nodes is assumed, i.e., $\theta_{\text {in }}=\phi_{\text {in }}=\phi_{\text {ro }}=0$.

Assume that the AUV oscillates around its centre-gravity in the three axes of $X_{t}, Y_{t}$, and $Z_{t}$, and model the corresponding displacements $\Delta_{x}, \Delta_{y}$, and $\Delta_{z}$ by independently distributed Gaussian random variables [39], i.e., $\left\{\Delta_{x}, \Delta_{y}\right\} \sim \mathcal{N}\left(0, \sigma_{x y}^{2}\right)$, and $\Delta_{z} \sim \mathcal{N}\left(-L_{o}, \sigma_{z}^{2}\right)$. Note that $\sigma_{z}$ and $\sigma_{x y}$ are the standard deviations of the link distance variations and off-axis misalignments, respectively.

In order to simplify capacity calculation, we consider two different scenarios, denoted by $S 3$ and $S 4$, where the $S 3$ assumes a fixed channel length (i.e., $\Delta_{z}=0$ ), and $S 4$ assumes on-axis transmission (i.e., $\Delta_{x}=\Delta_{y}=0$ ).

In case of scenario $S 3$, the average link capacity is calculated as 


$$
\bar{C}\left(\sigma_{x y}\right)=\int_{-\infty}^{\infty} \int_{-\infty}^{\infty} \frac{C(\boldsymbol{\Delta})}{2 \pi \sigma_{x y}^{2}} \exp \left(\frac{-\left(\Delta_{x}^{2}+\Delta_{y}^{2}\right)}{\left(2 \sigma_{x y}^{2}\right)}\right) d \Delta_{x} d \Delta_{y} .
$$

Also, in case of scenario $S 4$, the average link capacity is

$$
\bar{C}\left(\sigma_{z}\right)=\int_{-\infty}^{\infty} \frac{C(\boldsymbol{\Delta})}{\sqrt{2 \pi \sigma_{z}^{2}}} \exp \left(-\frac{\left(\Delta_{z}+L_{o}\right)^{2}}{\left(2 \sigma_{z}^{2}\right)}\right) d \Delta_{z}
$$

Both B2F and M2F links considered here have ranges less than $L_{t h}$ in Eq. (9) to guarantee high capacity communication unaffected by window truncation.

\section{Tracked Angular MiMO (TA-MIMO) Systems}

Given that link alignment between the transmitter and the receiver is a major challenge in practice, in this section, a PLT solution for the A-MIMO technique is presented, termed tracked A-MIMO (TA-MIMO). Leveraging the inherent geometric optics properties of A-MIMO systems, this PLT solution utilizes a $\left(D_{r} \sqrt{M_{r}} / \delta \times D_{r} \sqrt{M_{r}} / \delta\right)$ sub-pixel array to estimate rotation, shifting, and truncation of the received image. In the following subsections, the estimation of rotation, shifting and truncation of the received image are first introduced. Afterwards, the capacity of TA-MIMO links is calculated for the given distribution of tracking residual errors (TREs).

\section{A. Image Rotation and Shifting}

In the case of perfect beam alignment, the received image in an A-MIMO link is an inverted and distorted version of the transmitted image due to receiver lens and channel impairments, respectively. However, in the case of a misaligned link, the received image is additionally rotated or shifted relative to $\phi_{r_{o}}$ or $\left(\theta_{i n}, \phi_{i n}\right)$ angles. As shown in Fig. 4, a rotation in the optical axis of the transmitter by $\phi_{\text {ro }}$ leads to the rotation of the received image by the same value. Also, an inclination of the transmitter by $\left(\theta_{i n}, \phi_{i n}\right)$ causes in a shift in the centre of the received image from the origin of the PD array $(0,0)$ to a new position $\left(\dot{x}_{s h}, \dot{y}_{s h}\right)$. In the following, a classical image registration algorithm [40], [41] is adopted to estimate the rotation and inclination angles for the A-MIMO link. 
1) Rotation Angle: Assume that the transmitted image can be identified using pilot symbols, where the receiver can distinguish between the spots of the different LDs without ambiguity. Let $g_{o}\left(\dot{x}_{r}, \dot{y}_{r}\right)$ be the received "reference" image under perfect alignment, and $g_{r}\left(\dot{x}_{r}, \dot{y}_{r}\right)$ denote the received image at misaligned orientation. In the PLT scheme, the value of the rotation angle can be estimated using phase correlation between $g_{o}\left(\dot{x}_{r}, \dot{y}_{r}\right)$ and $g_{r}\left(\dot{x}_{r}, \dot{y}_{r}\right)$ in log-polar Fourier transform domain. Thus, $\phi_{r o}$ can be estimated regardless of the values of $\left(\theta_{i n}, \phi_{i n}\right)$ which reduces the TRE [40], [41].

To obtain the $\log$-polar Fourier transform, the magnitude spectrum of $g_{o}\left(\dot{x}_{r}, \dot{y}_{r}\right)$ and $g_{r}\left(\dot{x}_{r}, \dot{y}_{r}\right)$ are obtained by using fast Fourier transform (FFT) to yield $\left|G_{o}\left(w_{x}, w_{y}\right)\right|$ and $\left|G_{r}\left(w_{x}, w_{y}\right)\right|$, respectively. Then, the spectrum is converted to polar coordinates with log scale for the radius to yield $\left|G_{o}(\rho, \phi)\right|$ and $\left|G_{r}(\rho, \phi)\right|$. Finally, the spectrum in log-polar domain, $G_{r}\left(w_{\rho}, w_{\phi}\right)$ and $G_{o}\left(w_{\rho}, w_{\phi}\right)$, are calculated from $\left|G_{o}(\rho, \phi)\right|$ and $\left|G_{r}(\rho, \phi)\right|$ using FFT again. The estimate of rotation angle is obtained by calculating the phase correlation between $G_{r}\left(w_{\rho}, w_{\phi}\right)$ and $G_{o}\left(w_{\rho}, w_{\phi}\right)$ as [42]

$$
\hat{\phi}_{r o}=\arg \max _{\phi}: \mathbf{F}^{-1}\left\{\frac{G_{o}\left(w_{\rho}, w_{\phi}\right) G_{r}^{*}\left(w_{\rho}, w_{\phi}\right)}{\left|G_{o}\left(w_{\rho}, w_{\phi}\right)\right|\left|G_{r}^{*}\left(w_{\rho}, w_{\phi}\right)\right|}\right\},
$$

where, $\mathbf{F}^{-1}$ denotes inverse FFT. Equation (36) estimates $\phi_{\text {ro }}$ with $180^{\circ}$ ambiguity, that is, the estimated rotation value is either $\hat{\phi}_{r o}$ or $\hat{\phi}_{r o}+\pi$. Therefore, the phase correlation between the reference image and the received one with rotations $\hat{\phi}_{r o}$ and $\hat{\phi}_{r o}+\pi$ should be compared; the rotated image associated to the larger phase correlation will be the correct image [40]. The resulting image from this step is named "compensated" image $g_{c}\left(\dot{x}_{r}, \dot{y}_{r}\right)$, which is used in the next step.

2) Inclination Angles: After estimating and compensating the rotational misalignment, the image shift due to inclination can be also estimated using cross correlation between the original and compensated images as in [41]. As a result, $\left(\dot{x}_{s h}, \dot{y}_{s h}\right)$ can be estimated as [42]

$$
\left(\hat{\dot{x}}_{s h}, \hat{\dot{y}}_{s h}\right)=\arg \max _{\dot{x}_{r}, \dot{y}_{r}}: \mathbf{F}^{-\mathbf{1}}\left\{G_{o}\left(w_{x}, w_{y}\right) G_{c}^{*}\left(w_{x}, w_{y}\right)\right\}
$$

where $G_{o}\left(w_{x}, w_{y}\right)$ and $G_{c}^{*}\left(w_{x}, w_{y}\right)$ are FFT and conjugate FFT in the Cartesian coordinates for the original image and the compensated one, respectively. Geometrically, as illustrated in Fig. 4, the inclination angles can be calculated using estimated values $\left(\hat{\dot{x}}_{s h}, \hat{\dot{y}}_{s h}\right)$ and Eq. (6) as 


$$
\begin{aligned}
& \hat{\theta}_{i n}=\arctan \left(\frac{\hat{r}_{s h}}{f_{r}}\right), \hat{r}_{s h}=\sqrt{\hat{\dot{x}}_{s h}^{2}+\hat{\dot{y}}_{s h}^{2},} \\
& \hat{\phi}_{i n}= \begin{cases}\arccos \left(\hat{\dot{x}}_{s h} / \hat{r}_{s h}\right), & \text { if } \quad \hat{\dot{y}}_{s h}<0, \\
\arccos \left(\hat{\dot{x}}_{s h} / \hat{r}_{s h}\right)+\pi, & \text { if } \quad \hat{\dot{y}}_{s h} \geq 0 .\end{cases}
\end{aligned}
$$

Using Eqs. (36)-(38), the orientation of the TA-MIMO link can be tracked and maintained.

\section{B. Image Truncation}

As discussed in Section II, in A-MIMO links the received image is not truncated as long as $L \leq L_{t h}$. Under this condition, since the system magnification is fixed, the PLT scheme is not able to estimate the channel length or off-axis deviation. However, when $L>L_{t h}$, the received image is truncated by a circle of area $A_{w}$ at centre $\left(\dot{x}_{c}, \dot{y}_{c}\right)$, which can be estimated (e.g., using [43]) and used to infer the off-axis shift and distance between the transmitter and the receiver.

Given the estimated area for the received image, $\hat{A}_{w}$, the channel length can be estimated as [10]

$$
\hat{L}=\pi \times \frac{\left(f_{r}\left(l_{r}+l_{t}\right)\right)}{\sqrt{4 / \pi \hat{A}_{w}}}, \quad \hat{L} \geq L_{t h} .
$$

Using the estimated centre $\left(\hat{\dot{x}}_{c}, \hat{\dot{y}}_{c}\right)$ and the channel length, the shift values in the transmitter axes can be estimated by [44]

$$
\hat{\Delta}_{z}=\hat{L}-L_{o}, \quad \hat{\Delta}_{x}=\frac{\hat{L} \hat{\dot{x}}_{c}}{f_{r}}, \quad \hat{\Delta}_{y}=\frac{\hat{L} \hat{\dot{y}}_{c}}{f_{r}} .
$$

Using Eqs. (39) and (40), the on-axis alignment and the channel length of TA-MIMO link can be tracked.

Figure 6 presents a flowchart summarizing the PLT scheme, which concurrently estimates the orientation and the off-axis misalignments. In order to consider TREs, we define the vector $\Delta_{T R E}$ as

$$
\begin{gathered}
\Delta_{T R E}=\left[\theta_{e} ; \phi_{e_{i}} ; \phi_{e_{r}} ; x_{e} ; y_{e} ; z_{e}\right], \\
\theta_{e}=\left|\theta_{i n}-\hat{\theta}_{i n}\right|, \quad \phi_{e_{i}}=\left|\phi_{i n}-\hat{\phi}_{i n}\right|, \quad \phi_{e_{r}}=\left|\phi_{r o}-\hat{\phi}_{r o}\right|, \\
x_{e}=\left|\Delta_{x}-\hat{\Delta}_{x}\right|, \quad y_{e}=\left|\Delta_{y}-\hat{\Delta}_{y}\right|, \quad z_{e}=\Delta_{z}-\hat{\Delta}_{z} .
\end{gathered}
$$




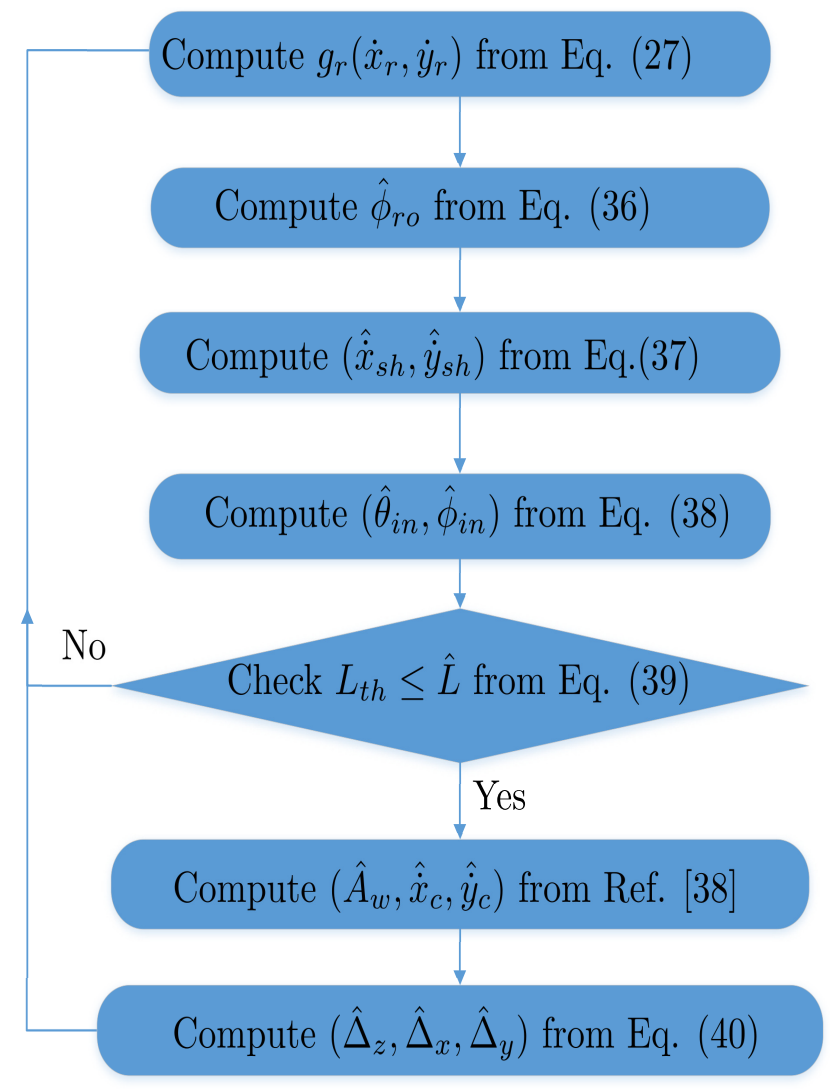

Fig. 6. Flowchart of the proposed PLT scheme for TA-MIMO systems.

one disadvantage of this approach is that the TREs will largely depend on the resolution of the imaging receiver (i.e., the number of sub-PDs per PD array). In addition, small TREs in $\phi_{\text {ro }}$ and $L$ may lead to relatively larger TREs in $\left(\theta_{i n}, \phi_{i n}\right)$ and $\left(\Delta_{x}, \Delta_{y}\right)$, respectively. These tradeoffs are further investigated through numerical simulations in Section VI.

\section{Capacity of TA-MIMO links}

For the B2F communication model, the average capacity of TA-MIMO link is calculated as

$$
\bar{C}(U)=\int_{0}^{2 \pi} \int_{0}^{\pi / 2} C\left(\boldsymbol{\Delta}_{T R E}\right) p_{\theta_{e}, \phi_{e_{\chi}}}\left(\theta_{e}, \phi_{e_{\chi}}\right) d \theta_{e} d \phi_{e_{\chi}}
$$

where $p_{\theta_{e}, \phi_{e_{\chi}}}\left(\theta_{e}, \phi_{e_{\chi}}\right)$ is the joint PDF of TREs, and $\phi_{e_{\chi}}$ equals $\phi_{e_{r}}$ and $\phi_{e_{i}}$ for scenarios $S 1$ and $S 2$, respectively. Note that, $p_{\theta_{e}, \phi_{e_{\chi}}}\left(\theta_{e}, \phi_{e_{\chi}}\right)$ is calculated using Eqs. (27), (32) and (36)-(41). The accuracy of $p_{\theta_{e}, \phi_{e_{\chi}}}\left(\theta_{e}, \phi_{e_{\chi}}\right)$ will be later verified via numerical simulations in Section VI. 
TABLE II

PARAMETERS OF A-MIMO AND TA-MIMO Links [16], [45]

\begin{tabular}{|c|c|c|}
\hline \multicolumn{3}{|c|}{ Transmitter parameters } \\
\hline Diameter of the lens $\left(l_{t}\right)$ & \multicolumn{2}{|c|}{$0.1 \mathrm{~m}$} \\
\hline Focal length of the lens $\left(f_{t}\right)$ & \multicolumn{2}{|c|}{$0.365 \mathrm{~m}$} \\
\hline Magnification scale $\left(M_{A}\right)$ & \multicolumn{2}{|c|}{1} \\
\hline No. of LDs $\left(M_{t}\right)$ & \multicolumn{2}{|c|}{49} \\
\hline Distance between the centres of the LDs $\left(D_{t}\right)$ & \multicolumn{2}{|c|}{$5 \mathrm{~mm}$} \\
\hline Average transmitted optical power of each LD $\left(P_{o}\right)$ & \multicolumn{2}{|c|}{$20.41 \mathrm{~mW}$} \\
\hline LD wavelength $(\lambda)$ & \multicolumn{2}{|c|}{$532 \mathrm{~nm}$} \\
\hline Waist of Gaussian beam $\left(w_{o}\right)$ & \multicolumn{2}{|c|}{$0.075 \mathrm{~mm}$} \\
\hline Divergence angle of Gaussian beam & \multicolumn{2}{|c|}{$2.25 \mathrm{mrad}$} \\
\hline \multicolumn{3}{|l|}{ Receiver parameters } \\
\hline Diameter of the lens $\left(l_{r}\right)$ & \multicolumn{2}{|c|}{$0.5 \mathrm{~m}$} \\
\hline No. of PDs $\left(M_{r}\right)$ & \multicolumn{2}{|c|}{49} \\
\hline Distance between the centres of the PDs $\left(D_{r}\right)$ & \multicolumn{2}{|c|}{$5 \mathrm{~mm}$} \\
\hline Field of view of PD $\left(\theta_{F o V}\right)$ & \multicolumn{2}{|c|}{$180^{\circ}$} \\
\hline Responsitivity of PD ( $\Re)$ & \multicolumn{2}{|c|}{$0.8 \mathrm{~A} / \mathrm{W}$} \\
\hline Receiver Gaussian noise variance $\left(\sigma_{n}^{2}\right)$ & \multicolumn{2}{|c|}{$10^{-6} \mathrm{~A}^{2}$} \\
\hline Resolution of the imaging receiver $(\delta)$ & \multicolumn{2}{|c|}{$0.49 \mathrm{~mm}$} \\
\hline Area of the imaging receiver (i.e., PD array) & \multicolumn{2}{|c|}{$35 \times 35$} $\mathrm{~mm}^{2}$ \\
\hline Channel parameters & Clear water & Coastal water \\
\hline Water absorption coefficient $(a)$ & $0.069 \mathrm{~m}^{-1}$ & $0.088 \mathrm{~m}^{-1}$ \\
\hline Water scattering coefficient $(b)$ & $0.08 \mathrm{~m}^{-1}$ & $0.216 \mathrm{~m}^{-1}$ \\
\hline Water extinction coefficient $(c)$ & $0.149 \mathrm{~m}^{-1}$ & $0.304 \mathrm{~m}^{-1}$ \\
\hline Average cosine of scattering $(g)$ & \multicolumn{2}{|c|}{0.919} \\
\hline Nominal channel length $\left(L_{o}\right)$ & \multicolumn{2}{|c|}{$5 \mathrm{~m}$} \\
\hline
\end{tabular}

For the M2F communication model, the inherent advantages of the A-MIMO approach do not require a tracking system when only displacement misalignments are present, as will be shown in Section VI.

\section{NUMERICAL RESULTS}

In this section, we provide numerical results for AoA, PoA and TRE distributions. Also, we evaluate the capacity of a $49 \times 49$ link using A-MIMO, TA-MIMO and C-MIMO techniques for $\mathrm{B} 2 \mathrm{~F}$ and M2F communication scenarios.

The main parameters used in the simulations are summarized in Table II. Both clear and coastal seawaters are considered with a nominal channel length of $L_{o}=5 \mathrm{~m}$ which is less than the window truncation threshold of $L_{t h}=6 \mathrm{~m}$. This channel length is less than the associated 
mean path length ${ }^{4}$ of the multiple scattering [46] in clear and coastal seawaters, which equals 13.5 and $6.6 \mathrm{~m}$, respectively. Here, it is assumed that the parameters of the clear and coastal seawaters (i.e., $a, b$ and $g$ ) are fixed with the seawater depth (for instance, see [47]). To make a fair comparison between A-MIMO, TA-MIMO, and C-MIMO systems, the same array parameters (i.e., $M_{t}, D_{t}, M_{r}, D_{r}$, and $l_{r}$ ) are used with identical LDs and PDs, as specified in Table II and described in the next subsection.

\section{A. A-MIMO and TA-MIMO Link Setup}

Figure 7 shows the layout of the considered A-MIMO and TA-MIMO transmitters. A $7 \times 7$ LD array with inter spacing of $5 \mathrm{~mm}$ is considered, with diameter and focal length of transmitter lens set to $l_{t}=0.10 \mathrm{~m}$ and $f_{t}=0.365 \mathrm{~m}$, respectively. The transmitted power from each LD is $P_{o}=20.41 \mathrm{~mW}$, giving a total transmit power ${ }^{5}$ of $P_{t}=1 \mathrm{~W}$. Each LD is considered to have a Gaussian beam profile with small beam waist and beam width to satisfy the assumptions of Section III (i.e., $w_{o} \ll D_{t}$ and $w \ll l_{t}$ ). The launching polar and azimuthal angles are associated to the position of LDs, as shown in the figure. On the receiver side, a $7 \times 7$ PD array is considered with inter spacing of $5 \mathrm{~mm}$, with the receiver lens of diameter $l_{r}=0.5 \mathrm{~m}$ and focal length $f_{r}=0.365 \mathrm{~m}$. Not that, although a receiver lens with diameter $0.5 \mathrm{~m}$ is relatively large, it is chosen here to illustrate the potential capacity increases with underwater optical MIMO systems corrupted by severe misalignment conditions ${ }^{6}$. For relatively less severe misalignment conditions, as considered in [13], [15], a smaller receiver lens can be used. For the case of TA-MIMO, the PD array is oversampled with the resolution $\delta=0.49 \mathrm{~mm}$ which means that a $71 \times 71$ sub-pixel array is considered for the tracking purposes.

\footnotetext{
${ }^{4}$ The mean path length is obtained by averaging $\mu$ using the PDF in Eq. (11), and it is equal to $1 / c$ and $2 / c$ for the single and multiple scattering, respectively.

${ }^{5}$ Though eye-safety is not explicitly considered here or in the related literature, it is an important feature of any wireless optical system. In the atmospheric wireless optical communications, eye-safety limits are well specified and depend strongly on wavelength and beam shape [48]. In practice, in underwater systems, large scattering, absorption and beam divergence would likely provide a more relaxed optical power limit at typical operating ranges.

${ }^{6}$ (e.g., off-axis up to 0.3 meter and wind speed up to 6 meter/second, resulting in polar inclination angle up to $10^{\circ}$ in the following)
} 


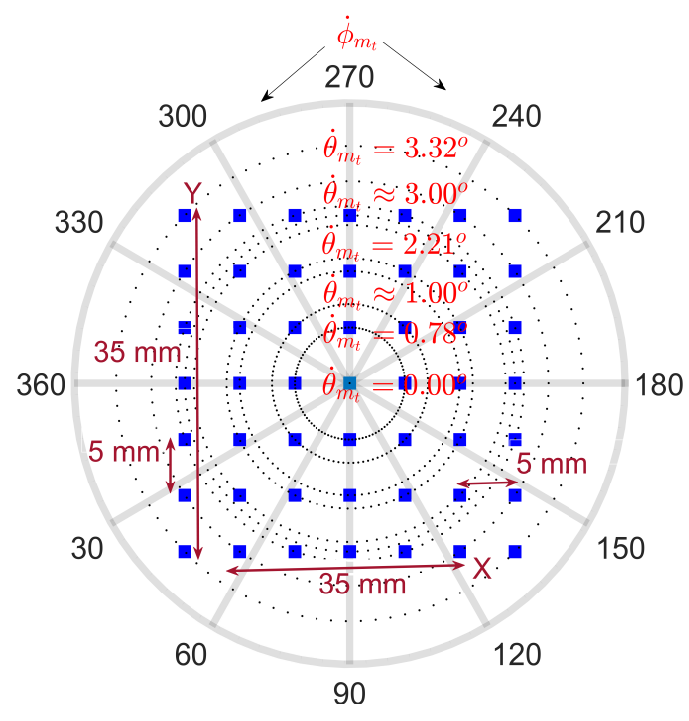

Fig. 7. Space-to-angle mapping $\left(\dot{\theta}_{m_{t}}, \dot{\phi}_{m_{t}}\right)$ for a $7 \times 7 \mathrm{LD}$ array of the A-MIMO and TA-MIMO transmitters.

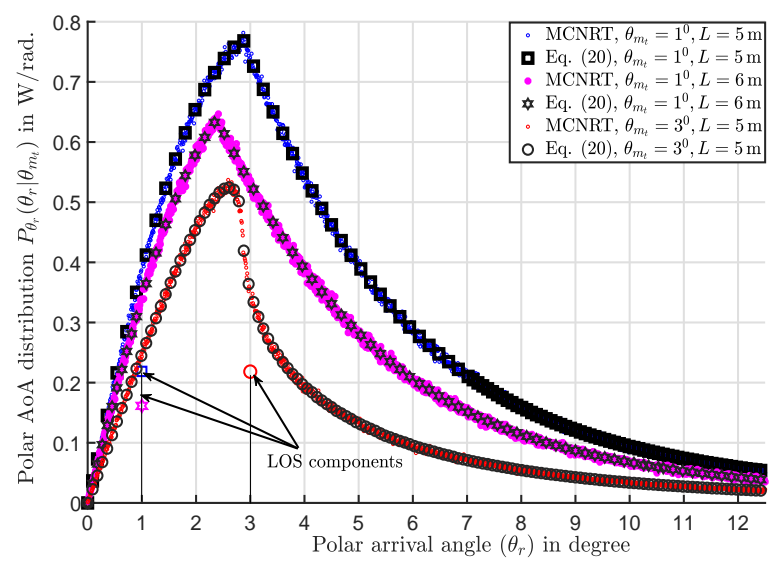

Fig. 8. Polar AoA distributions (obtained using Eq. (20)) in coastal seawaters with three settings for the transmitted polar angle $\left(\theta_{m_{t}}\right)$ and link length $(L)$.

\section{B. Results of Polar and Azimuthal AoA Distributions}

Figures 8-10 illustrate the polar and azimuthal AoA distributions in $\mathrm{W} / \mathrm{rad}$ for the A-MIMO and TA-MIMO links. The LOS component in the figure is not superimposed with single scattering component to show its absolute intensity. The results are obtained analytically ( using Eqs. (20)(23)) and verified through MCNRT simulations (Eqs. (11)-(13)) with the number of samples equal to $N_{s}=10^{6}$. Figure 8 shows polar AoA distributions calculated using Eq. (20) for the case of coastal seawaters with three settings of; (i) $\theta_{m_{t}}=1^{o}, L=5 \mathrm{~m}$ (ii) $\theta_{m_{t}}=3^{o}, L=5 \mathrm{~m}$ 


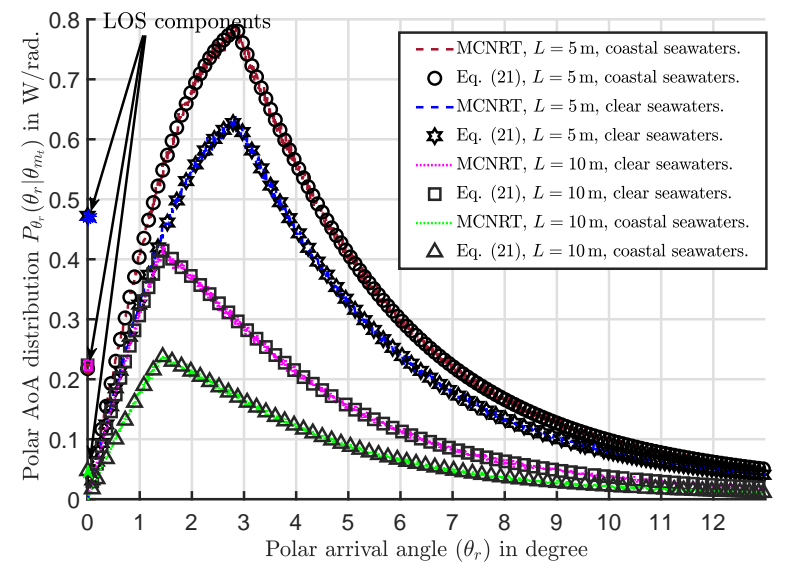

Fig. 9. Polar AoA distributions with $\theta_{m_{t}}=0^{\circ}$ (obtained using Eq. (21)) in clear and coastal seawaters and for link lengths $L=\{5,10\} \mathrm{m}$.

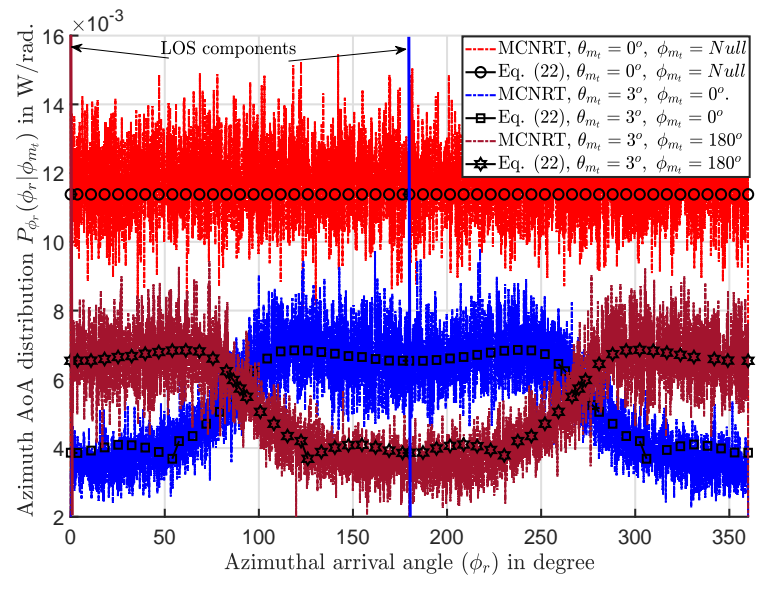

Fig. 10. Azimuthal AoA distributions (obtained by Eq. (22)) in coastal seawaters at nominal channel length of $5 \mathrm{~m}$ with three settings for polar and azimuthal transmitted angles.

and (iii) $\theta_{m_{t}}=1^{\circ}, L=6 \mathrm{~m}$. In these settings, the amplitude of the LOS component, as well as peak and dispersion of the distributions decrease with increase in the launching angle and the channel length. Numerically, the amplitude of LOS components are equal to $0.23,0.23$, and $0.18 \mathrm{~W} / \mathrm{rad}$, and the PDF peaks are equal to $0.77,0.52$, and $0.65 \mathrm{~W} / \mathrm{rad}$ for settings $(i)$, (ii), and $($ iii), respectively. Figure 9 shows polar AoA distributions at zero polar launching angle $\left(\theta_{m_{t}}=0\right)$ calculated using Eq. (21) for clear and coastal seawaters with channel lengths $L=5$ and $10 \mathrm{~m}$. The effect of seawater type on the LOS component, and peak and dispersion of the distribution can be observed. As expected, the power of the LOS component is larger in clear 

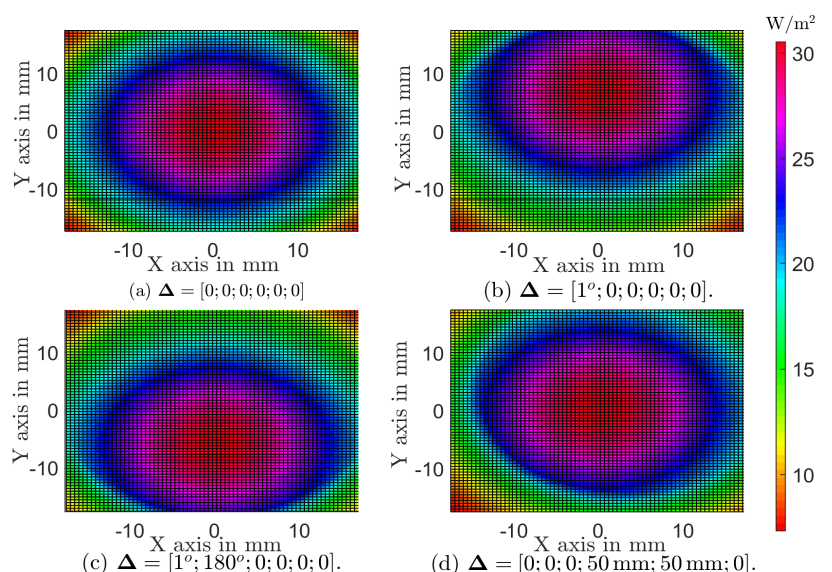

Fig. 11. PoA distributions (obtained from Eq. (24) with $\dot{\theta}_{m_{t}}=0$ ) in coastal seawaters at nominal channel length of $5 \mathrm{~m}$ with four misalignment conditions (a)-(d).

seawaters, as compared to coastal seawaters. Furthermore, the peak and the dispersion of the distribution are smaller for the case of clear seawaters. Numerically, the amplitude of the LOS components equal 0.47 and $0.21 \mathrm{~W} / \mathrm{rad}$, and the peaks equal 0.63 and $0.78 \mathrm{~W} / \mathrm{rad}$, for clear and coastal seawaters, respectively, at the nominal channel length of $L=5 \mathrm{~m}$.

Figure 10 shows the azimuthal AoA distributions, calculated using Eq. (22) for coastal seawaters with the nominal channel length of $5 \mathrm{~m}$ and for three settings of: $(i) \theta_{m_{t}}=0^{\circ}$, (ii) $\theta_{m_{t}}=3^{\circ}, \phi_{m_{t}}=0^{\circ}$, and $(i i i) \theta_{m_{t}}=3^{\circ}, \phi_{m_{t}}=180^{\circ}$. For setting $(i)$, the received distribution is uniform with the value $12 \times 10^{-3} \mathrm{~W} / \mathrm{rad}$, while the approximate expression of Eq. (23) gives $9 \times 10^{-3} \mathrm{~W} / \mathrm{rad}$. For settings $($ ii $)$ and (iii), the corresponding LOS and scattering components are equal due to the symmetry of the transmitter, with peaks around $7 \times 10^{-3} \mathrm{~W} / \mathrm{rad}$. Note that, the distributions of settings $(i i)$ and $(i i i)$ are relatively lower compared with setting $(i)$, which due to a higher geometric loss for the latter. Lastly, we notice from Figs. 8-10 that a good agreement between analytical and MCNRT simulation-based results.

\section{PoA and TRE Distributions}

Figures 11, 12 and 13 illustrate the PoA distribution, the TRE, and the TRE distribution for the TA-MIMO link, respectively. Also, the main results of these figures are summarized in Table III.

The PoA distributions in Figure 11 are obtained by using Eq. (24) for the central LD $\left(\dot{\theta}_{m_{t}}=\right.$ 0 ) in coastal seawaters with the nominal channel length. Four conditions are considered: $(a)$ Perfect alignment $(\boldsymbol{\Delta}=[0 ; 0 ; 0 ; 0 ; 0 ; 0]),(b)$ Polar angle misalignment $\left(\boldsymbol{\Delta}=\left[1^{o} ; 0 ; 0 ; 0 ; 0 ; 0\right]\right)$, 


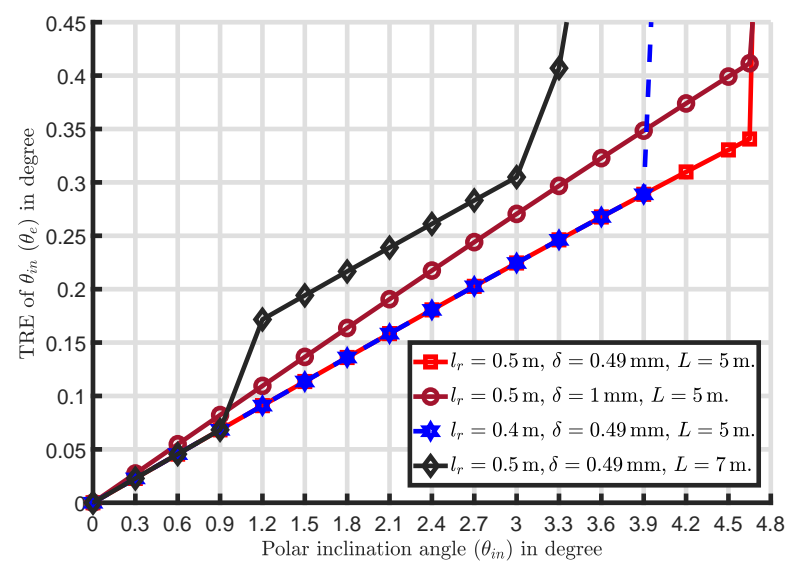

Fig. 12. Instance TRE of the polar inclination angle $\left(\theta_{e}\right.$, obtained by Eq. (41)) in coastal seawaters with four scenarios for $l_{r}$, $\delta$ and $L$.

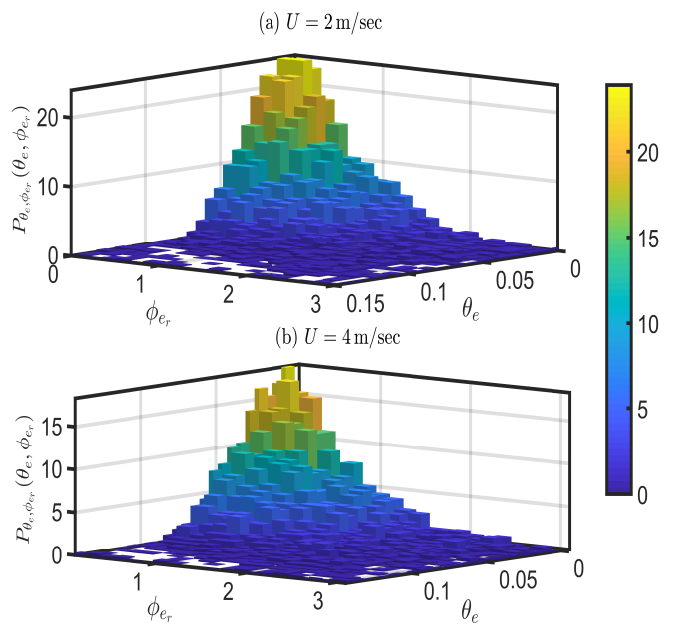

Fig. 13. Joint probability distributions of $\operatorname{TRE}\left(p_{\theta_{e}, \phi_{e_{r}}}\left(\theta_{e}, \phi_{e_{r}}\right)\right)$ for B2F-S1 scenario at two wind speeds: (a) $U=2 \mathrm{~m} / \mathrm{sec}$ and (b) $U=4 \mathrm{~m} / \mathrm{sec}$.

(c) Azimuthal angle misalignment $\left(\boldsymbol{\Delta}=\left[1^{o} ; 180^{\circ} ; 0 ; 0 ; 0 ; 0\right]\right)$, and $(d)$ Off-axis misalignment $(\boldsymbol{\Delta}=[0 ; 0 ; 0 ; 50 \mathrm{~mm} ; 50 \mathrm{~mm} ; 0])$, corresponding to sub-figures (a), (b), (c) and (d), respectively. Notice that the received image is shifted as a result of polar and azimuthal misalignment, as given in Eq. (38). In case of the off-axis misalignment, the received image endures minimal changes as a consequence of space-to-angle mapping. Note that, the small difference between the cases of (a) and (d) is mainly due to slight difference between the scattering and geometric loss conditions for these cases. 
TABLE III

MARGINAL PROBABILITY DISTRIBUTIONS OF TRE FOR B2F-S1 SCENARIO AT THREE WIND SPEEDS

\begin{tabular}{|c|c|c|c|}
\hline \multirow{2}{*}{ Parameters of $p_{\theta_{e}}\left(\theta_{e}\right)$ and $p_{\phi_{e_{r}}}\left(\phi_{e_{r}}\right)$} & \multicolumn{4}{|c|}{ Wind speed (U) in m/sec } \\
& 2 & 4 & 6 \\
\hline Mean of $\theta_{e}$ & $0.08^{\circ}$ & $0.12^{o}$ & $0.34^{o}$ \\
\hline Mode of $\theta_{e}$ & $0.03^{\circ}$ & $0.03^{\circ}$ & $0.1^{o}$ \\
\hline Standard deviation of $\theta_{e}$ & $0.23^{\circ}$ & $0.25^{\circ}$ & $1.05^{\circ}$ \\
\hline Standard error in mean of $\theta_{e}$ & $\pm 0.0045^{\circ}$ & $\pm 0.0049^{\circ}$ & $\pm 0.0207^{\circ}$ \\
\hline Mean of $\phi_{e_{r}}$ & $4^{o}$ & $6.75^{\circ}$ & $10.04^{\circ}$ \\
\hline Mode of $\phi_{e_{r}}$ & $1^{o}$ & $2^{o}$ & $2^{o}$ \\
\hline Standard deviation of $\phi_{e_{r}}$ & $10.23^{\circ}$ & $12.32^{\circ}$ & $13.5^{\circ}$ \\
\hline Standard error in mean of $\phi_{e_{r}}$ & $\pm 0.202^{\circ}$ & $\pm 0.2434^{\circ}$ & $\pm 0.266^{\circ}$ \\
\hline
\end{tabular}

Figure 12 shows the TRE $\theta_{e}$ at a given inclination misalignment angle $\theta_{i n}$ in coastal seawaters. The results are obtained by using Eq. (41) with $\boldsymbol{\Delta}=\left[\theta_{i n} ; 0 ; 0 ; 0 ; 0 ; 0\right]$. As shown, four scenarios are considered; $(i) l_{r}=0.5 \mathrm{~m}, \delta=0.49 \mathrm{~mm}, L=5 \mathrm{~m}$, (ii) $l_{r}=0.5 \mathrm{~m}, \delta=1 \mathrm{~mm}, L=5 \mathrm{~m}$, (iii) $l_{r}=0.4 \mathrm{~m}, \delta=0.49 \mathrm{~mm}, L=5 \mathrm{~m}$, and (iv) $l_{r}=0.5 \mathrm{~m}, \delta=0.49 \mathrm{~mm}, L=7 \mathrm{~m}$. Scenario (i) has the largest lens size, the best resolution (i.e., the lowest value for $\delta$ ), and the smallest channel length. Thus, this scenario achieves the best performance, i.e., the lowest TRE, among all other scenarios, as can be seen in the figure. The impact of receiver aperture size is apparent by comparing scenarios $(i)$ and (iii). Notice that the larger $l_{r}$ is, the greater is the range of inclinations $\theta_{i n}$ that can be estimated since aperture truncation is relaxed as it can be seen from Eq. (9). Figure 13 and Table III show respectively the joint and marginal PDFs of TRE for scenario $S 1$ of the B2F model. The distributions are computed using MCNRT method (Eqs. (27), (32) and (36)-(41)) under the assumption $\Delta=\left[\theta_{i n} ; 0 ; \phi_{r o} ; 0 ; 0 ; 0\right]$ with the number of samples of $N_{s}=16000$. The uncertainty due to the relatively small $N_{s}$ is bounded in Table III with upper and lower $99 \%$ confidence intervals [49].

In Fig. 13, the joint PDFs of TRE are evaluated at two wind speeds of $U=2$ and $4 \mathrm{~m} / \mathrm{sec}$. As shown, $p_{\theta_{e}, \phi_{e_{r}}}\left(\theta_{e}, \phi_{e_{r}}\right)$ approaches 2-D negative exponential PDF, where, reasonably, the mean and variance at $U=4 \mathrm{~m} / \mathrm{sec}$ are higher than those at $U=2 \mathrm{~m} / \mathrm{sec}$. To better see the impact of the wind speed on TRE, Table III presents means, modes, (i.e., peaks) and standard deviations of $p_{\theta_{e}}\left(\theta_{e}\right)$ and $p_{\phi_{e_{r}}}\left(\phi_{e_{r}}\right)$ distributions for three wind speeds of $U=\{2,4,6\} \mathrm{m} / \mathrm{sec}$. Notice that these parameters increase with $U$. For example, there is a factor of 4 difference between these values for $U=6 \mathrm{~m} / \mathrm{sec}$ and $U=2 \mathrm{~m} / \mathrm{sec}$. Also, the mean and mode parameters of $p_{\phi_{e_{r}}}\left(\phi_{e_{r}}\right)$ at 


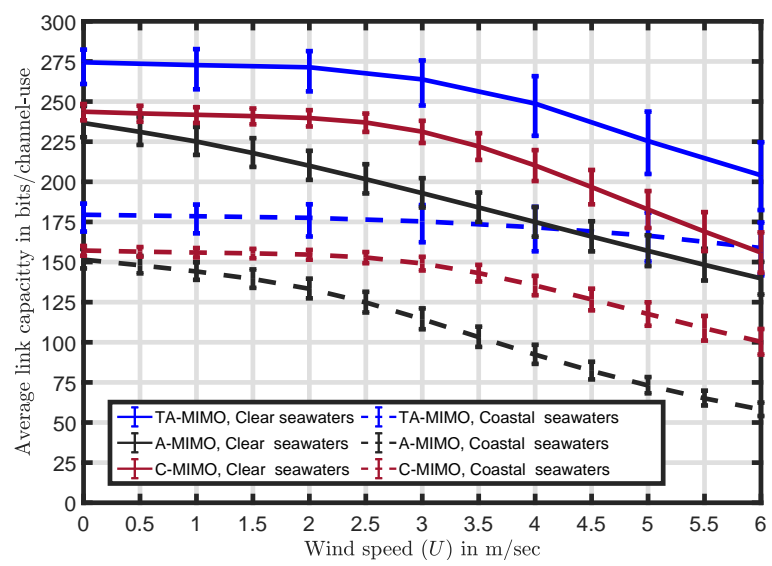

Fig. 14. Average capacity versus wind speed for scenario of B2F-S1 in clear and coastal seawaters with the nominal channel length of $5 \mathrm{~m}$.

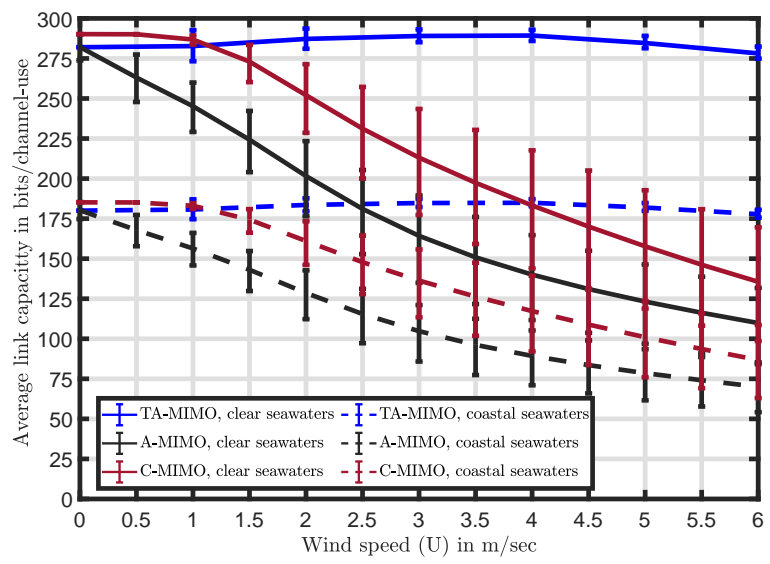

Fig. 15. Average capacity versus wind speed for scenario of B2F-S2 in clear and coastal seawaters with the nominal channel length (i.e. $L=L_{o}$ ).

$U=6 \mathrm{~m} / \mathrm{sec}$ are almost twice those at $U=2 \mathrm{~m} / \mathrm{sec}$.

\section{Link Capacity for B2F and M2F Models}

Figures 14-17 show the link capacity of $49 \times 49$ A-MIMO, TA-MIMO and C-MIMO systems for B2F and M2F communication models. The capacity is calculated using Eqs. (33)-(35) and (42), which are approximated through numerical integration. Errors between the actual and approximate values are bounded according to upper and lower Riemann sum theory [50], as shown by error bars in the figures. Under perfect alignment conditions, i.e., $\boldsymbol{\Delta}=[0 ; 0 ; 0 ; 0 ; 0 ; 0]$, 


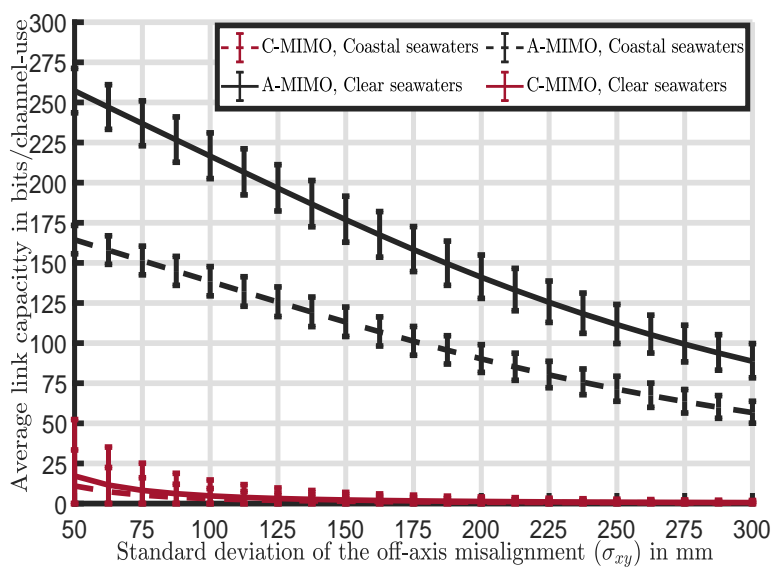

Fig. 16. Average capacity versus off-axis misalignment standard deviation for scenario M2F- $S 3$ in clear and coastal seawaters with the nominal channel length of $5 \mathrm{~m}$.

all links achieve roughly the same capacity of 290 and 185 bits/channel-use in clear and coastal seawaters, respectively.

For the B2F communication model, Figs. 14 and 15 show the capacity performance versus the wind speed $(U=[0,6] \mathrm{m} / \mathrm{sec})$ in scenarios $S 1$ and $S 2$, respectively. As shown, the capacity is degraded with increase in the wind speed, causing more orientation misalignments. As expected, the TA-MIMO system gives the best performance, whereas the lowest capacity is achieved for the A-MIMO system. In fact, for A-MIMO and C-MIMO links, the performance degrades faster with wind speed in the case of scenario $S 2$, compared with $S 1$. For the TA-MIMO link, the performance is nearly unchanged in the case of scenario $S 2$ relative to $S 1$. This may be interpreted by the fact that a small TRE $\phi_{e_{r}}$ leads to a relatively large TRE $\theta_{e}$, as mentioned in Section V. For instance, from Fig. 14, TA-MIMO, C-MIMO, and A-MIMO systems achieve capacity values of $263 \pm 13,230 \pm 5$ and $185 \pm 13$ bits/channel-use, respectively, at $U=3 \mathrm{~m} / \mathrm{sec}$ in clear seawaters. In coastal seawaters, these capacity values decrease to $175 \pm 7,150 \pm 5$ and $113 \pm 10$ bits/channel-use, respectively. Also, from Fig. 15, TA-MIMO, C-MIMO, and A-MIMO systems achieve capacity values of $285 \pm 5,213 \pm 5$ and $163 \pm 12$ bits/channel-use, respectively, at $U=3 \mathrm{~m} / \mathrm{sec}$ in clear seawaters. For coastal seawaters, these capacity values decrease to $180 \pm 5$, $137 \pm 5$ and $110 \pm 12$ bits/channel-use, respectively. 


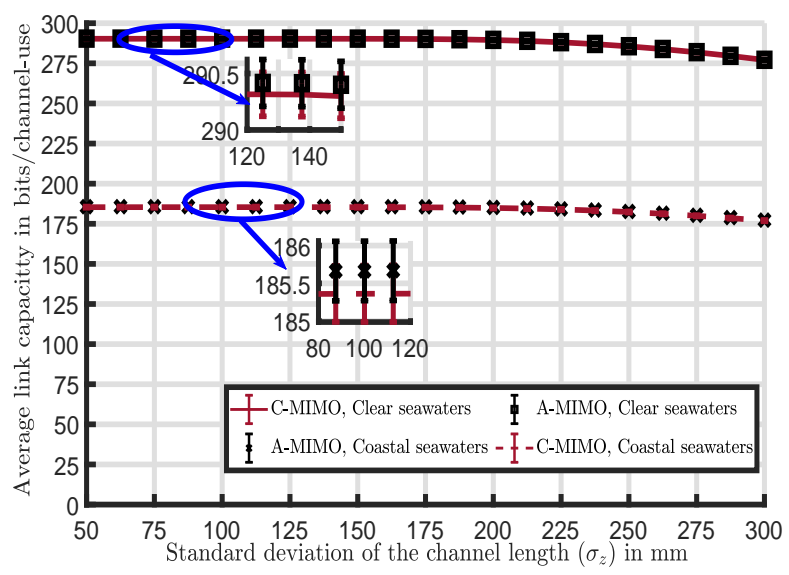

Fig. 17. Average capacity versus channel length standard deviation for scenario of M2F-S4 in clear and coastal seawaters (with mean channel length $L_{o}$ )

For the M2F communication model ${ }^{7}$, Figs. 16 and 17 compare the capacity of A-MIMO and C-MIMO systems for scenarios $S 3$ and $S 4$, respectively. Figure 16 shows the capacity versus standard deviation of the off-axis misalignment, $\sigma_{x y}$. Notice that at $\sigma_{x y}=75 \mathrm{~mm}$, the A-MIMO system achieves capacity values $237 \pm 25$ and $150 \pm 7$ bits/channel-use in clear and coastal seawaters, respectively, whereas the corresponding C-MIMO capacity values are much lower, i.e., $14 \pm 12.5$ and $10 \pm 9$ bits/channel-use. As well, at off-axis misalignment of $300 \mathrm{~mm}$, the A-MIMO system achieves capacity values $55 \pm 12.5$ and $80 \pm 12.5$ bits/channel-use in clear and coastal seawaters, respectively, whereas the corresponding C-MIMO capacity values are $1 \pm .5 \mathrm{bit} / \mathrm{channel-use}$. Note additionally that the capacity decrease for A-MIMO link is due to the increased geometric loss and not due to the off-axis misalignment. These results clearly show that A-MIMO outperforms C-MIMO due to its inherent angle-to-space mapping. Figure 17 shows the capacity performance versus standard deviation of the channel length $\sigma_{z}$. The capacity performance is almost fixed for $\sigma_{z} \leq 225 \mathrm{~mm}$. However, there is a drop of nearly 13 bits/channel-use at $\sigma_{z}=300 \mathrm{~mm}$ with respect to the fixed channel length case, i.e., $\sigma_{z}=0$. In short range applications, as considered here, the performances of A-MIMO and C-MIMO links are roughly the same in the case of perfect on-axis alignment (i.e., $\sigma_{x y}=0$ ). However, the A-MIMO system slightly outperforms C-MIMO, as shown in the enlarged plots. Lastly, note that

\footnotetext{
${ }^{7}$ Note that, no numerical results are presented for TA-MIMO in cases of the M2F scenarios, where $L_{o}<L_{t h}$ and no misalignment parameter can be inferred at that length as shown in Fig. 6.
} 
here we considered scenarios S3 and S4 in order to show the impact of off-axis misalignment and channel length variations independently. However, in a realistic scenario, a M2F system will be displaced in the three axes (i.e. off-axis and length variation) concurrently. The link performance in this realistic scenario can be inferred from the results of Figs. 16 and 17 together.

\section{CONCLUSIONS AND Discussions}

In this paper, A-MIMO technique was proposed for short-range high-speed underwater applications, which is more robust against axes-displacement in contrast to C-MIMO technique. The architecture of A-MIMO results in a simple PLT scheme to yield the TA-MIMO system, which estimates the link misalignment. Additionally, a comprehensive link modelling was presented for A-MIMO and TA-MIMO, which is quite useful for the system design.

The presented numerical results demonstrated that C-MIMO generally outperform A-MIMO in the case where angular misalignment dominate, such as those prevalent in the B2F scenario. However, by using the angular information present, the TA-MIMO system can improve over CMIMO to give high channel capacities. The A-MIMO system, however, greatly outperforms CMIMO when off-axis misalignment dominate (e.g., in the M2F scenario). By sending information in angular domain, the A-MIMO system is robust against off-axis shifts, and the corresponding capacity remains relatively insensitive to small variations in the channel length.

In realistic scenarios, where both angular and off-axis displacements are present, the choice between A-MIMO and C-MIMO systems depends on the link misalignment conditions. For instance, if the link displacement dominates the misalignment, A-MIMO was shown here to be a good approach to achieve a higher capacity performance. In addition, TA-MIMO is a good choice when both angular misalignment and off-axis displacements are present, so long as the added complexity is tolerable.

The challenges of the implementation of A-MIMO/TA-MIMO systems include the transceiver size, which must be carefully chosen depending on the communication range and the link misalignment. The TA-MIMO system requires a high-resolution PD array, which is associated with the accuracy of the estimation for the relative misalignment between the ends of the link. Lastly, like C-MIMO systems, A-MIMO/TA-MIMO systems are not appropriate solutions in severe scattering channels (i.e., high turbidity seawaters) due to the incurred inter-channel interference. 


\section{ACKNOWLEDGEMENTS}

The authors would like to thank Mr. Khaqan Majeed and Mr. Tau Rasethuntsa for their efforts in proofreading the manuscript.

\section{APPENDIX A}

PROOFS OF EQUATIONS (19), (21) AND (23)

\section{A. Proof of Equation (19)}

Assuming that the beam spot on the transmitter lens is relatively small $\left(w \ll l_{t}\right)$, the $m_{t}^{\text {th }}$ LD can be well approximated as a single-ray source. According to this assumption, the double integration over $\left(x_{t}, y_{t}\right)$ in Eq. (18), i.e., $\int_{x_{t}} \int_{y_{t}} I_{m_{t}}\left(x_{t}, y_{t}\right) d y_{t} d x_{t}$, is reduced to the transmitted optical power $P_{o}$.

Moreover, the integration over $\mu_{0}$ in Eq. (18) can be solved for the central LD $\left(x_{t}=0, y_{t}=0\right)$ with on-axis alignment assumption $\left(\Delta_{x}=0, \Delta_{y}=0\right)$ as follows. The geometric loss function $G_{m_{t}}\left(x_{r}^{s}, y_{r}^{s}\right)$ depends on $\mu_{0}$ as indicated in Eqs. (14), (16) and (17). Thus, the inequality in Eq. (14) can be rewritten in terms of $\mu_{0}$ using Eqs. (16) and (17) assuming: $x_{t}=y_{t}=\Delta_{x}=\Delta_{y}=0$. The solution of $\mu_{0}$ lies in the range $\mu_{s_{1}} \leq \mu_{0} \leq \mu_{s_{2}}$, where $\mu_{s_{1}}$ and $\mu_{s_{2}}$ are given as

$$
\begin{aligned}
& \left\{\mu_{s_{1}}\left(\theta_{r}, \phi_{r}\right), \mu_{s_{2}}\left(\theta_{r}, \phi_{r}\right)\right\}= \\
& \quad \frac{f_{3}\left(\theta_{r}, \phi_{r}\right)}{2 f_{4}\left(\theta_{r}, \phi_{r}\right)} \pm \sqrt{\left[\frac{f_{3}\left(\theta_{r}, \phi_{r}\right)^{2}}{4 f_{4}\left(\theta_{r}, \phi_{r}\right)^{2}}-\frac{L^{2} \tan \left(\theta_{r}\right)^{2}-l_{r}^{2} / 4}{f_{4}\left(\theta_{r}, \phi_{r}\right)}\right]},
\end{aligned}
$$

where functions $f_{3}\left(\theta_{r}, \phi_{r}\right)$ and $f_{4}\left(\theta_{r}, \phi_{r}\right)$ are defined as

$$
\begin{aligned}
f_{3}\left(\theta_{r}, \phi_{r}\right)= & 2 L\left[\sin \left(\phi_{m_{t}}\right) \sin \left(\phi_{r}\right)+\cos \left(\phi_{m_{t}}\right) \cos \left(\phi_{r}\right)\right] \\
& \times \tan \left(\theta_{r}\right) \sin \left(\theta_{m_{t}}\right)-2 L \tan \left(\theta_{r}^{2}\right) \cos \left(\theta_{m_{t}}\right), \\
f_{4}\left(\theta_{r}, \phi_{r}\right)= & \sin \left(\theta_{m_{t}}\right)^{2}+\tan \left(\theta_{r}\right)^{2} \cos \left(\theta_{m_{t}}\right)^{2}-2 \tan \left(\theta_{r}\right) \\
& \times \cos \left(\phi_{r}-\phi_{m_{t}}\right) \sin \left(\phi_{m_{t}}\right) \cos \left(\phi_{m_{t}}\right) .
\end{aligned}
$$

The common range between the limits of the integration over $\mu_{o}$, i.e., $0 \leq \mu_{0} \leq L / \cos \left(\theta_{t}\right)$, and $\mu_{s_{1}} \leq \mu_{0} \leq \mu_{s_{2}}$ can be defined by two optimization functions as follows:

$$
\begin{aligned}
& f_{1}\left(\theta_{r}, \phi_{r}\right)=\max \left\{0, \mu_{s_{1}}\left(\theta_{r}, \phi_{r}\right)\right\}, \\
& f_{2}\left(\theta_{r}, \phi_{r}\right)=\min \left\{\frac{L}{\cos \left(\theta_{t}\right)}, \mu_{s_{2}}\left(\theta_{r}, \phi_{r}\right)\right\} .
\end{aligned}
$$


Therefore, integrating the first term of Eq. (18) over $f_{1}\left(\theta_{r}, \phi_{r}\right) \leq \mu_{0} \leq f_{2}\left(\theta_{r}, \phi_{r}\right)$ yields a closed-form AoA distribution for the central LD with on-axis alignment as shown in Eq. (19).

\section{B. Proof of Equation (21)}

Equation (20) can be rewritten in a simpler form with zero launching angle, i.e., $\theta_{m_{t}}=0$. By substituting $\theta_{m_{t}}=0$ in Eq. (43), the solutions of the inequality and the scattering angle are obtained as

$$
\begin{aligned}
& \left\{\mu_{s_{1}}\left(\theta_{r}\right), \mu_{s_{2}}\left(\theta_{r}\right)\right\}= \\
& {\left[-\left(L+l_{r} \cot \left(\theta_{r}\right) / 4\right),\left(l_{r} \cot \left(\theta_{r}\right) / 4-L\right)\right],} \\
& \theta_{s}=\theta_{r} .
\end{aligned}
$$

Thus, the integration over $\phi_{r}$ in Eq. (20) can be computed analytically as shown in Eq. (21).

\section{Proof of Equation (23)}

In case of the central $\operatorname{LD}\left(x_{t}=0, y_{t}=0\right)$ at on-axis transmission $\left(\Delta_{x}=0, \Delta_{y}=0\right)$ and zero launching angle $\left(\theta_{m_{t}}=0\right)$, the azimuthal AoA distribution is uniform as shown in Fig. 10, and is computed as

$$
P_{\phi_{r}}\left(\phi_{r} \mid \theta_{m_{t}}=0\right)=\frac{P_{r}}{2 \pi}
$$

where $P_{r}$ is the total received power on the receiver lens which is obtained by integrating Eq. (21) over $\theta_{r}$ using the following integral forms [51]

$$
\begin{aligned}
& \int \exp \left[-a x^{2}\right] d x=\sqrt{\frac{\pi}{4 a}} \operatorname{erf}(\sqrt{a} x), \\
& \int x^{m} \exp \left[-\beta x^{n}\right] d x=\frac{1}{\left(n \beta^{\gamma}\right)} \Gamma\left(\gamma, n \beta x^{n}\right),
\end{aligned}
$$

where $\gamma=(m+1) / n$ and $\{\beta, n\} \neq 0$. The ingratiation in Eq. (48) is calculated using the following approximations

$$
\begin{gathered}
{\left[\frac{\sin \left(\theta_{r}\right)\left(\sec \left(\theta_{r}\right)-1\right)^{-1}}{\left(2+2 g^{2}-4 g \cos \left(\theta_{r}\right)\right)^{3 / 2}}\right] \approx\left[156.3 \theta_{r}^{-1.519}-1802\right]} \\
\sec \left(\theta_{r}\right) \approx\left[\theta_{r}^{2} / 2+1\right]
\end{gathered}
$$


The first approximation in Eq. (49) is done at $g=0.91$ by using curve fitting tool (i.e. cftool tool box) in Matlab [18]. The second one is done by using Taylor series expansion. So, the approximated closed-form is given in Eq. (23).

\section{REFERENCES}

[1] Z. Zeng, S. Fu, H. Zhang, Y. Dong, and J. Cheng, “A survey of underwater optical wireless communications,” IEEE Communications Surveys Tutorials, vol. 19, no. 1, pp. 204-238, Firstquarter 2017.

[2] M. A. Khalighi, C. J. Gabriel, L. M. Pessoa, and B. Silva, Visible Light Communications: Theory and Applications. CRC-Press, 2017, ch. Underwater Visible Light Communications, Channel Modeling and System Design, pp. $337-372$.

[3] K. Nakamura, I. Mizukoshi, and M. Hanawa, "Optical wireless transmission of 405 nm, 1.45 Gbit/s optical IM/DD-OFDM signals through a 4.8 m underwater channel," Opt. Express, vol. 23, no. 2, pp. 1558-1566, Jan 2015. [Online]. Available: http://www.opticsexpress.org/abstract.cfm?URI=oe-23-2-1558

[4] P. Zou, Y. Zhao, F. Hu, and N. Chi, "Underwater visible light communication at 3.24 Gb/s using novel two-dimensional bit allocation," Opt. Express, vol. 28, no. 8, pp. 11319-11338, Apr 2020. [Online]. Available: http://www.opticsexpress.org/abstract.cfm?URI=oe-28-8-11319

[5] H. Kaushal and G. Kaddoum, "Underwater optical wireless communication,” IEEE Access, vol. 4, pp. 1518-1547, 2016.

[6] M. A. Khalighi, H. Akhouayri, and S. Hranilovic, "Silicon-photomultiplier-based underwater wireless optical communication using pulse-amplitude modulation," IEEE Journal of Oceanic Engineering, vol. 45, no. 4, pp. 1611-1621, Octuber 2020.

[7] N. Saeed, S. Guo, K.-H. Park, T. Y. Al-Naffouri, and M.-S. Alouini, "Optical camera communications: Survey, use cases, challenges, and future trends," Phyisical Communications, vol. 37, Dec. 2019.

[8] S. Hranilovic and F. R. Kschischang, "A pixelated MIMO wireless optical communication system," IEEE Journal of Selected Topics in Quantum Electronics, vol. 12, no. 4, pp. 859-874, July 2006.

[9] L. Zeng, D. C. O’Brien, H. L. Minh, G. E. Faulkner, K. Lee, D. Jung, Y. Oh, and E. T. Won, "High data rate multiple input multiple output (MIMO) optical wireless communications using white Led lighting," IEEE Journal on Selected Areas in Communications, vol. 27, no. 9, pp. 1654-1662, Dec. 2009.

[10] B. Han and S. Hranilovic, "A fixed-scale pixelated MIMO visible light communication system," IEEE Journal on Selected Areas in Communications, vol. 36, no. 1, pp. 203-211, Jan 2018.

[11] Y. Dong, J. Liu, and H. Zhang, "On capacity of 2-by-2 underwater wireless optical MIMO channels," in 2015 Advances in Wireless and Optical Communications (RTUWO), Riga, Nov 2015, pp. 219-222.

[12] H. Zhang, Y. Dong, and L. Hui, "On capacity of downlink underwater wireless optical MIMO systems with random sea surface," IEEE Communications Letters, vol. 19, no. 12, pp. 2166-2169, Dec. 2015.

[13] H. Zhang, J. Cheng, Z. Wang, and Y. Dong, "On the capacity of buoy-based MIMO systems for underwater optical wireless links with turbulence," in 2018 IEEE International Conference on Communications (ICC), Kanses City, MO, May 2018, pp. 1-6.

[14] Y. Li, H. Qiu, X. Chen, J. Fu, M. Musa, and X. Li, "Spatial correlation analysis of imaging MIMO for underwater visible light communication," in Optics Communications, Elsevier, vol. 443, no. 4, Jul. 2019, pp. 221-229.

[15] R. Xu, Y. Chen, Z. Wei, H. Fu, J. Cheng, and Y. Dong, "On BER of fixed-scale MIMO underwater wireless optical communication systems," in 2020 IEEE 17th Annual Consumer Communications Networking Conference (CCNC), Las Vegas, NV, USA, 2020, pp. 1-6. 
[16] A. S. Ghazy, S. Hranilovic, and M. A. Khalighi, "Angular MIMO for underwater wireless optical communications: Channel modelling and capacity," in 2019 16th Canadian Workshop on Information Theory (CWIT), Hmailotn, Ontario, Canada, June 2019, pp. 1-6.

[17] “Zemax opticstudio," https://www.zemax.com, accessed: 2019-11-14.

[18] "Matlab: MCNRT and curve fitting," https://www.mathworks.com, accessed: 2019-11-14.

[19] M. Pisani, "Four quadrant photo-detector with ultra high common mode rejection ratio and ultra narrow gap," in The 17th Annual Meeting of the IEEELasers and Electro-Optics Society, 2004. LEOS 2004, Rio, Grande, Pureto Rico, vol. 1, 2004, pp. 51-52 Vol.1.

[20] A. Mohan, G. Woo, S. Hiura, Q. Smithwick, and R. Raskar, "Bokode: Imperceptible visual tags for camera based interaction from a distance," ACM Trans. Graph., vol. 28, no. 3, pp. 98:1-98:8, July 2009. [Online]. Available: http://doi.acm.org/10.1145/1531326.1531404

[21] G. F. Simmons, Calculus with Analytic Geometry. New York : McGraw-Hill., 1996.

[22] H. Zhang and Y. Dong, "General stochastic channel model and performance evaluation for underwater wireless optical links," IEEE Transactions on Wireless Communications, vol. 15, no. 2, pp. 1162-1173, Feb. 2016.

[23] C. Gabriel, M. A. Khalighi, S. Bourennane, P. Leon, and V. Rigaud, "Monte-carlo-based channel characterization for underwater optical communication systems," IEEE/OSA Journal of Optical Communications and Networking, vol. 5, no. 1, pp. 1-12, Jan. 2013.

[24] B. Cochenour, L. Mullen, and J. Muth, "Temporal response of the underwater optical channel for high-bandwidth wireless laser communications," IEEE Journal of Oceanic Engineering, vol. 38, no. 4, pp. 730-742, Oct. 2013.

[25] G. Xu and J. Lai, "Average capacity analysis of the underwater optical plane wave over anisotropic moderate-to-strong oceanic turbulence channels with the malaga fading model," Opt. Express, vol. 28, no. 16, pp. 24 056-24 068, Aug. 2020.

[26] "Gaussian beam optics," [Online]. 2020-01-04. Available: https://web.pa.msu.edu/courses/2010fall/phy431/PostNotes/PHY431-Notes-GaussianBeamOptics.pdf

[27] R. A. Leathers, T. V. Downes, C. O. Davis, C. D. Mobley, R. A. Leathers, T. V. Downes, C. O. Davis, and C. D. Mobley, "Monte carlo radiative transfer simulations for ocean optics: A practical guide," Tech. Rep., 2004.

[28] J. Thompson and W. O. Popoola, "Effects of turbulence induced scattering on underwater optical wireless communications," arXiv preprint arxiv:2008.01152, 2020.

[29] J. L. Henyey and L. G. Greenstein, "Diffuse radiation in the galaxy," in Astrophysical Journal, Jan. 1941, pp. 70-83.

[30] J. S. L. and W. L., Monte Carlo Modeling of Light Transport in Tissues. Boston, MA: Springer US, 1995, pp. 73-100.

[31] T. Hamza, M.-A. Khalighi, S. Bourennane, P. Léon, and J. Opderbecke, "Investigation of solar noise impact on the performance of underwater wireless optical communication links," in in Opt. Express, vol. 24, no. 22, pp. 25832-25845, Oct. 2016.

[32] S. Tang, Y. Dong, and X. Zhang, "Impulse response modeling for underwater wireless optical communication links," IEEE Transactions on Communications, vol. 62, no. 1, pp. 226-234, Jan. 2014.

[33] G. J. Foschini and M. J. Gans, "On limits of wireless communications in a fading environment when using multiple antennas," Wireless Personal Communications, vol. 6, no. 3, pp. 311-335, 1996.

[34] H. M. Oubei, J. R. Duran, B. Janjua, H.-Y. Wang, C.-T. Tsai, Y.-C. Chi, T. K. Ng, H.-C. Kuo, J.-H. He, M.-S. Alouini, G.-R. Lin, and B. S. Ooi, "4.8 Gbit/s 16-QAM-OFDM transmission based on compact 450-nm laser for underwater wireless optical communication," Opt. Express, vol. 23, no. 18, pp. 23 302-23 309, Sep. 2015.

[35] J. Xu, Y. Song, X. Yu, A. Lin, M. Kong, J. Han, and N. Deng, "Underwater wireless transmission of high-speed QAMOFDM signals using a compact red-light laser," Opt. Express, vol. 24, no. 8, pp. 8097-8109, Apr. 2016. 
[36] C. Albaladejo, F. Soto, R. Torres, P. Sánchez, and J. A. López, "A low-cost sensor buoy system for monitoring shallow marine environments," Sensors, vol. 12, no. 7, pp. 9613-9634, 2012.

[37] C. Cox and W. Munk, "Measurement of the roughness of the sea surface from photographs of the sun's glitter," J. Opt. Soc. Am., vol. 44, no. 11, pp. 838-850, Nov 1954.

[38] I. Vasilescu, K. Kotay, D. Rus, M. Dunbabin, and P. Corke, "Data collection, storage, and retrieval with an underwater sensor network," in Proceedings of the 3rd International Conference on Embedded Networked SensorSystems, San Diego, Californiam USA, 2005, pp. 154-165.

[39] T. I. Fossen, Guidance and Control of Ocean Vehicles. Wiley, 1994.

[40] B. S. Reddy and B. N. Chatterji, "An FFT-based technique for translation, rotation, and scale-invariant image registration," IEEE Transactions on Image Processing, vol. 5, no. 8, pp. 1266-1271, Aug. 1996.

[41] C. A. Wilson and J. A. Theriot, "A correlation-based approach to calculate rotation and translation of moving cells," IEEE Transactions on Image Processing, vol. 15, no. 7, pp. 1939-1951, July 2006.

[42] G. Tzimiropoulos, V. Argyriou, S. Zafeiriou, and T. Stathaki, "Robust FFT-based scale-invariant image registration with image gradients," IEEE Transactions on Pattern Analysis and Machine Intelligence, vol. 32, no. 10, pp. 1899-1906, Oct 2010.

[43] E. E. Zelniker and I. V. L. Clarkson, "Maximum-likelihood estimation of circle parameters via convolution," IEEE Transactions on Image Processing, vol. 15, no. 4, pp. 865-876, Apr. 2006.

[44] B. Han, “A fixed-scale pixelated MIMO visible light,” Master's thesis, McMaster University, 2017.

[45] C. Mobley, "Light and water: Radiative transfer in natural waters," in San Diego, CA: Academic Press/Elsevier Science, Jan. 1994, pp. 249-519.

[46] J. Thompson and W. O. Popoola, "Effects of turbulence induced scattering on underwater optical wireless communications," arXiv preprint arXiv:2008.01152, 2020.

[47] L. J. Johnson, R. J. Green, and M. S. Leeson, "Underwater optical wireless communications: depth dependent variations in attenuation," Appl. Opt., vol. 52, no. 33, pp. 7867-7873, Nov. 2013.

[48] "Laser products and instruments," https://www.fda.gov/radiation-emitting-products/home-business-and-entertainmentproducts/laser-products-and-instruments, accessed: 2020-8-7.

[49] G. J. HAHN, "Sample sizes for monte carlo simulation," IEEE Transaction n Systems, Man, and Cybernetics, vol. ???, no. ???, pp. 219-222, Nov 1972.

[50] H. Bear, "A primer of lebesgue integration," in Academic Press, Sep. 2002, pp. 249-519.

[51] D. Zwillinger, V. Moll, I. Gradshteyn, and I. Ryzhik, “Table of integrals, series, and products," in Academic Press, Oct. 2015, pp. 249-519. 


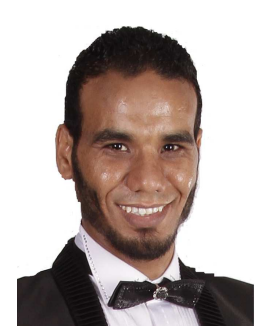

Abdallah S. Ghazy was born in Giza, Egypt, in 1983. He received the B.S. degree in electrical engineering from Azher University, Egypt, in 2007. From 2008 to 2016, he has worked in communication systems in Telecome-Egypt and Avaya business partner companies, Cairo, Egypt. In 2012, he joined the electrical engineering school, Azher University, Egypt. He received the M.S degree in electrical engineering from Egypt-Japan University of Science and Technology, Alexandria, Egypt, in 2016. He is currently on leave from the Azher University and is working toward the Ph.D. degree, electrical and computer engineering school, Mcmaster University, Ontario, Canada. His main research interests include optical communications, wireless communications and digital signal processing.

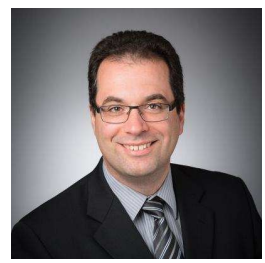

STEVE HRANILOVIC (Senior Member, IEEE) received the B.A.Sc. degree (Hons.) in electrical engineering from the University of Waterloo, Canada, in 1997, and the M.A.Sc. and Ph.D. degrees in electrical engineering from the University of Toronto, Canada, in 1999 and 2003, respectively. He is a Professor with the Department of Electrical and Computer Engineering, McMaster University, Hamilton, ON, Canada, and currently serves as the Associate Dean (Academic). From 2010 to 2011, he spent his research leave as a Senior Member, Technical Staff in Advanced Technology for Research in Motion, Waterloo, ON, Canada. He has authored the book Wireless Optical Communication Systems (New York: Springer, 2004). His research interests are in the areas of free-space and optical wireless communications, digital communication algorithms, and electronic and photonic implementation of coding and communication algorithms.

Dr. Hranilovic was awarded the Government of Ontario Early Researcher Award in 2006. In 2016, the title of University Scholar was conferred upon him by McMaster University. He is a Licensed Professional Engineer in the Province of Ontario. He has served as an Associate Editor for the Journal of Optical Communications and Networking and an Editor for the IEEE TRANSACTIONS ON COMMUNICATIONS in the area of Optical Wireless Communications.

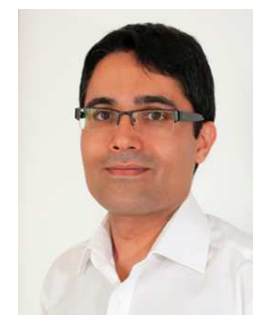

MOHAMMAD ALI KHALIGHI (Senior Member, IEEE) is Associate Professor with École Centrale Marseille, Marseille, France, and head of "Optical Communications for IoT" group at Fresnel Institute research lab. He is currently serving as Project Coordinator for the H2020 ITN MSCA VisIoN project (Visible-light-based Interoperability and Networking) and Action Chair for the COST Action CA19111 NEWFOCUS (European Network on Future Generation Optical Wireless Communication Technologies). He has coedited the book "Visible Light Communications: Theory and Applications" (CRC Press, 2017) and was the co-recipient of the 2019 Best Survey Paper Award of the IEEE Communications Society. He is also serving as Editor-at-Large for the IEEE Transactions on Communications, and served as Associate Editor for the IET Electronics Letters as well as Guest Editor for the IEEE Open Journal of the Communications Society and Elsevier Optik journal. His main research interests include signal processing for wireless communication systems with an emphasis on the physical layer aspects of freespace, underwater, and indoor visible-light optical communications. 